YITP-96-9

February 29, 1996

\title{
The spectral representation of the spacetime structure: The 'distance' between universes with different topologies
}

\author{
Masafumi Seriu \\ Inter-University Centre for Astronomy and Astrophysics \\ Post Bag 4, Ganeshkhind, Pune 41100\%, India \\ and \\ Yukawa Institute for Theoretical Physics \\ Kyoto University, Kyoto 606, Japan ${ }^{\star}$
}

To appear in Physical Review D.

\footnotetext{
$\star$ Present address.
} 


\begin{abstract}
We investigate the representation of the geometrical information of the universe in terms of spectra, i.e. a set of eigenvalues of the Laplacian defined on the universe. Here, we concentrate only on one specific problem along this line: To introduce a concept of distance between universes in terms of the difference in the spectra.

We can find out such a measure of closeness from a general discussion: First, we introduce a suitable functional $P_{\mathcal{G}}[\cdot]$, where the geometrical information $\mathcal{G}$ (represented by the spectra) determines the detailed shape of the functional. Then, the overlapping functional integral between $P_{\mathcal{G}}[\cdot]$ and $P_{\mathcal{G}^{\prime}}[\cdot]$ is taken, providing a measure of closeness between $\mathcal{G}$ and $\mathcal{G}^{\prime}, d\left(\mathcal{G}, \mathcal{G}^{\prime}\right)$.

The basic properties of this distance (hereafter referred to as 'spectral distance', for brevity) are then investigated. First, it can be related to a reduced density matrix element in quantum cosmology between $\mathcal{G}$ and $\mathcal{G}^{\prime}$. Thus, calculating the spectral distance $d\left(\mathcal{G}, \mathcal{G}^{\prime}\right)$ gives us an insight for the quantum theoretical decoherence between two universes, corresponding to $\mathcal{G}$ and $\mathcal{G}^{\prime}$. Secondly, the spectral distance becomes divergent except for when $\mathcal{G}$ and $\mathcal{G}^{\prime}$ have the same dimension and volume. This is very suggestive if the above-mentioned density-matrix interpretation is taken into account. Thirdly, $d\left(\mathcal{G}, \mathcal{G}^{\prime}\right)$ does not satisfy the triangular inequality, which illustrates clearly that the spectral distance and the distance defined by the DeWitt metric on the superspace are not equivalent.
\end{abstract}


We then pose a question: Whether two universes with different topologies interfere with each other quantum mechanically? In particular, we concentrate on the difference in the orientabilities. To investigate this problem, several concrete models in 2-dimension are set up, and the spectral distances between them are investigated: Distances between tori and Klein's bottles, and those between spheres and real projective spaces. Quite surprisingly, we find many cases of spaces with different orientabilities in which the spectral distance turns out to be very short. It may suggest that, without any other special mechanism, two such universes interfere with each other quite strongly, contrary to our intuition.

We discuss some curious features of the heat kernel for tori and Klein's bottles in terms of Epstein's theta and zeta functions. Differences and parallelisms between the spectral distance and the DeWitt distance are also discussed.

\section{Introduction}

There are many situations in spacetime physics in which space/spacetime topology takes part. First of all, it is a central problem of modern cosmology to determine whether our universe is open or closed (i.e. non-compact or compact) [1] and what kind of topological structure our universe possesses [2],[3],[4]. The so-called worm-holes (topological handles attached to the universe) can cause many interesting phenomena, e.g. geons [5], charge without charge [6], time-machines [7]. The phenomenon of the topology change is one of the most intriguing problems in quantum cosmology [8].

Amongst these phenomena, let us now look at the scale-dependent topology (or physical topology) [9], [10] in more detail. Mathematically, topology can be looked upon as a global property of a manifold classified by the concept of continuity or continuous deformations [11]. Thus by definition, it is a scale-independent concept, i.e. objects with different scales, but continuously being deformed to each other are identified. 
However, once the concept of topology is applied to spacetime physics, the situation becomes different [10]: Because of the limitation in the observational energy scale, thin objects smaller than $E^{-1}$ cannot be observed ( $E$ : the energy scale of observation). Suppose the universe is topologically complicated, with two kinds of small topological handles ${ }^{\star}$ attached, one kind of scale $l$ and the other kind of scale $L, l \ll L$. If our observational energy is between $L^{-1}$ and $l^{-1}$, the smaller handles cannot be observed and the effective topology becomes simpler than the original one. If the energy scale is decreased further, such that it becomes less than $L^{-1}$, bigger handles also cannot be observed, resulting in a much simpler effective topology. Such a picture of spacetime structure, that the real universe is topologically very complicated, but the effective topology becomes simpler when the observational energy scale becomes lower, originates from Wheeler's spacetime foam picture [12]. In short, the scale-dependent topology (physical topology) can be looked upon as topology with distinction between big and small handles.

There is a clear lack of a suitable language to describe such phenomena sketched in the previous paragraph. To describe a sequential change in the effective topology of a space as a function of observational energy, we first need to formulate closeness between two spaces whose geometries are different globally as well as locally. There is no known mathematical theory suitable for this purpose. The aim of this paper is to formulate the concept of closeness between two spaces from the viewpoint of spacetime physics.

The main obstacle is the fact that we have to represent information of topology as well as local geometry in a quantitative, unified manner. Let us then pay attention to the idea of using the spectra to characterize the geometrical content of the universe: Basic vibrations (harmonics) of some matter field on the universe should reflect the local and global geometry of the universe. For definiteness, let $(\mathcal{M}, g)$ be a compact Riemannian manifold. Here $g$ is a positive definite metric.

* The term 'handle' usually means an object diffeomorphic to $D^{r} \times D^{n-r}(0 \leq r \leq n)$, where $D^{k}$ stands for $k$-dimensional disk, and $n$ is the dimension of a manifold. In this paper, however, any object which is topologically non-trivial is generally called 'handle'. 
Therefore, $(\mathcal{M}, g)$ is regarded either the spatial section of the $(n+1)$-dimensional universe, or the Euclidean $n$-dimensional universe. As the simplest elliptic operator, take the Laplace-Beltrami operator $\Delta=g^{a b} D_{a} D_{b}=\frac{1}{\sqrt{g}} \partial_{a}\left(\sqrt{g} g^{a b} \partial_{b}\right)$, where $D_{a}$ stands for the covariant derivative operator. Then, we can set up the eigenvalue problem on $(\mathcal{M}, g) ; \Delta \psi+\lambda \psi=0$ with a suitable boundary condition. For simplicity, let us assume $\partial \mathcal{M}=\emptyset$ here. Then, a set of eigenvalues is obtained; $\left\{\lambda_{k}\right\}, 0=\lambda_{0}<\lambda_{1} \leq \lambda_{2} \cdots \uparrow \infty$. This set of eigenvalues of the Laplacian clearly contains the information of both local and global geometry of $(\mathcal{M}, g)$. Hereafter, these eigenvalues of the Laplacian shall be called spectra, for brevity. We may be able to treat these spectra as a part of the fundamental quantities of spacetime physics.

The above idea of representing the geometrical content of the universe in terms of spectra is deeply related to a well-known mathematical problem, which is usually stated as 'Can one hear the shape of a drum?' [13], i.e. a problem asking as to what extent spectra reflect the underlying geometry. In this spirit, the motto of our approach may be stated as 'Let us hear the shape of the universe.' Here, the appearance of two terms 'hear' and 'shape' symbolizes the interaction between physics and mathematics. In short, our idea is to convert the space(time) structure (a mathematical object) into spectra or 'components of sound' (physical objects). This conversion may be called 'spectral representation' of the spacetime structure.

There are several advantages of such a representation. First, spectra represent the information of local and global geometry in an unified manner, which is suitable for applications of quantum gravity/cosmology. Secondly, the spectral representation is a representation of the geometry in terms of a countable set of real, positive numbers, which is easy to handle. Thirdly, spectra are the diffeomorphism invariant quantities, which is appropriate from the viewpoint of general relativity.

However, we should keep the following point in mind from the very beginning: There exist the isospectral manifolds, i.e. two Riemannian manifolds which are 
non-isometric to each other, but have identical spectra. Such a case has first been constructed by Milnor on $T^{16}$ [14], and several other cases have also been presented later $[15],[16],[17]$. Thus, in general, the information contained in geometry is larger than the one represented by spectra. It is uncertain, however, as to what extent these counter-examples are influential on spacetime physics. In any case, it is clear that the idea of the spectral representation is still worth while to pursue extensively.

In this spirit, we shall concentrate on one specific, tractable problem, which has been already implied above, i.e. the introduction of a measure of closeness between two universes of different geometrical structures.

In section 2, we shall construct a general theory of the 'spectral distance', i.e. a measure of closeness between two manifolds in terms of spectra. Then, the physical interpretation of this spectral distance shall be investigated. Afterwards, the necessary condition for the convergence of the spectral distance shall be studied. We shall also define a scale-dependent spectral distance, which provides a quantitative description of the scale-dependent topology. Finally, we shall discuss a peculiar property of the spectral distance, i.e. the failure of the triangular inequality. This result clearly illustrates the non-equivalence between the spectral distance and the distance defined by the DeWitt metric on the superspace [18].

In section 3, we shall pose a quantum cosmological question as to whether universes with different topologies (especially different orientabilities) interfere with each other quantum mechanically. We shall use the spectral distance as a suitable tool for analyzing this question. To investigate this problem, we shall construct concrete 2-dimensional models of $T^{2}$ and Klein's bottles, and those of $S^{2}$ and $\mathbf{R} P^{2}$. We shall then calculate the spectral distances between them. We shall find many cases in which the spectral distance between two manifolds of different orientabilities turns out to be surprisingly short. This result may imply that two universes with different orientabilities sometimes interfere quite strongly, contrary to our intuition. The DeWitt distances shall be also calculated for the cases of $T^{2}$ and they are compared with the spectral distances. We shall also construct and 
investigate the heat kernel for $T^{2}$ and Klein's bottles in terms of Epstein's theta and zeta functions [19],[20],[17].

In the final section, the spectral distance and the distance defined by the DeWitt metric shall be compared with each other in detail. Several other discussions shall also be presented.

\section{The spectral distance and its properties}

\subsection{THE SPECTRAL DISTANCE}

In this subsection, we shall search for the measure of closeness between two given manifolds. Let $(\mathcal{M}, g)$ be a $n$-dimensional Riemannian manifold. For clarity of discussion, we shall assume that $(\mathcal{M}, g)$ is compact and $g$ has the Riemannian signature, and not the pseudo-Riemannian signature. (Thus, $(\mathcal{M}, g)$ can be regarded as a mathematical model of the spatial section of the $(n+1)$-dimensional universe, or a model of the Euclidean $n$-dimensional universe. Hereafter, we may sometimes refer to $(\mathcal{M}, g)$ just as 'space' or 'universe', depending on the context.) The manifold $(\mathcal{M}, g)$ includes two types of information: local geometry and global topology. We shall use the term geometry in the broadest sense, including both. We shall also use the symbol $\mathcal{G}$ to represent the geometrical information contained in $(\mathcal{M}, g)$ in this broad sense.

Now, suppose two geometries $\mathcal{G}$ and $\mathcal{G}^{\prime}$ are given, corresponding to manifolds $(\mathcal{M}, g)$ and $\left(\mathcal{M}^{\prime}, g^{\prime}\right)$, respectively. Our goal is to find out a suitable formula for the 'distance' $d\left(\mathcal{G}, \mathcal{G}^{\prime}\right)$, representing a closeness between $\mathcal{G}$ and $\mathcal{G}^{\prime}$ in terms of the eigenvalues (hereafter spectra, for brevity ) of some elliptic differential operator $D$. From the viewpoint of physical applications, the most interesting and simplest choice is to take as $D$ the Laplace-Beltrami operator $\Delta, \Delta=g^{a b} D_{a} D_{b}=\frac{1}{\sqrt{g}} \partial_{a}\left(\sqrt{g} g^{a b} \partial_{b}\right)$, where $D_{a}$ stands for the covariant derivative operator. The basic idea for doing

* Another interesting choice as $D$ may be the Dirac operator. In this paper, however, let us confine ourselves to the case of the Laplacian, since it is more basic and well-investigated in geometry. 
this is as follows:

(1) Instead of comparing geometries directly (which is difficult), introduce a suitable functional $P_{\mathcal{G}}[\cdot]$, where the information of a geometry $\mathcal{G}$ enters into this functional as a parameter and reflects on the shape of the functional.

(2) By taking the overlapping functional integral between $P_{\mathcal{G}}[\cdot]$ and $P_{\mathcal{G}^{\prime}}[\cdot]$, we can indirectly measure the closeness of two geometries $\mathcal{G}$ and $\mathcal{G}^{\prime}$.

As a preliminary consideration, let us deal with functions instead of functionals: Suppose we need to introduce a concept of closeness in a set $\mathcal{S}=\{a, b, \cdots\}$. First we fix a function in which each element of $\mathcal{S}$ enters as a parameter, $\left\{p_{a}(\cdot), p_{b}(\cdot), \cdots\right\}$. (For instance, $\mathcal{S}$ is a set of positive numbers and $p_{a}(\cdot)$ is a Gaussian function with dispersion $a$.) Let us choose functions $p_{i}(\cdot)(i=a, b, \cdots)$ such that $p_{i}(\cdot) \geq$ 0 and $\int_{-\infty}^{\infty} p_{i}(x) d x=1$. In this case, a suitable overlapping integral is $\xi_{i j}=$ $\int_{-\infty}^{\infty}\left(p_{i}(x) p_{j}(x)\right)^{\frac{1}{2}} d x$, which has the properties,

(i) $\xi_{i j}=\xi_{j i}$.

(ii) $0 \leq \xi_{i j} \leq 1$, and

$$
\xi_{i j}=0 \Longleftrightarrow p_{i}(\cdot) p_{j}(\cdot) \equiv 0, \quad \xi_{i j}=1 \Longleftrightarrow p_{i}(\cdot) \equiv p_{j}(\cdot) .
$$

Thus, we can define a measure of closeness between ' $i$ ' and ' $j$ ' as

$$
d(i, j)=-\ln \xi_{i j}=-\ln \int_{-\infty}^{\infty}\left(p_{i}(x) p_{j}(x)\right)^{\frac{1}{2}} d x
$$

Then, $d(i, j)$ takes some value between 0 (complete overlapping) and $\infty$ (complete non-overlapping). Here ends this preliminary consideration.

There are several possibilities for the choice of $P_{\mathcal{G}}[\cdot]$ and the form of the overlapping functional integral. Here, we are mainly interested in the physical applications of the distance, so that we have to select a 'physically interesting' distance amongst other possibilities. 
First, we clearly need a distance $d\left(\mathcal{G}, \mathcal{G}^{\prime}\right)$ which is diffeomorphism invariant w.r.t. $\mathcal{G}$ and $\mathcal{G}^{\prime}$. As a simple functional which is diffeomorphism invariant, which reflects the global as well as the local geometry, and which includes the derivative operator (since it should be related to the spectra of a differential operator), we take $\sigma[\cdot]$ as $^{\star}$

$$
\sigma[f]:=\frac{1}{2} \int_{\mathcal{M}}(\partial f)^{2}=\frac{1}{2} \int_{\mathcal{M}} g^{a b} \partial_{a} f \partial_{b} f \sqrt{g} .
$$

Roughly speaking, in the above functional, the appearance of the metric $g$ reflects the local geometry, while the integral over $\mathcal{M}$ reflects the global geometry.

However, $\sigma[\cdot]$ is not enough for our purpose since it takes an arbitrarily large positive value so that it cannot be normalized. Thus, let us fix a suitable function $F(\cdot)$ such that $P[\cdot]:=F(\sigma[\cdot])$ is well-behaved. In view of the preliminary consideration above, $P[\cdot]$ should satisfy

(I) $P[\cdot] \geq 0, \quad \int[d f] P[f]=1$.

Furthermore, we shall need a physically sensible 'distance' applicable to the analysis of the scale-dependent topology, which requires that smaller handles should be ignored under certain conditions, compared with larger handles. Thus,

(II) The higher (lower) weight for the longer (shorter) scale behavior of $\mathcal{G}$.

There are still various possibilities that remain for the choice of $F(\cdot)$. Here, for several reasons, we shall choose one possibility amongst many others, $F(x)=$ $\exp -x$ : First, it is a simple function which is easy to handle. Secondly, it makes us possible to relate the distance with a physical concept, i.e. the quantum decoherence of the universes, which shall be discussed in detail in the next subsection. Thirdly, it satisfies the requirements (I) and (II): Consider the eigenvalue problem $\Delta \phi_{k}+\lambda_{k} \phi_{k}=0$ (with a suitable boundary condition; mainly the case of $\partial \mathcal{M}=\emptyset$ shall be described here.) Thus we get the spectra $0=\lambda_{0}<$

$\star$ We can also choose as $\sigma[f]:=\frac{1}{2} \int_{\mathcal{M}}\left\{(\partial f)^{2}+\mu^{2} f^{2}\right\}$. Here, $\mu$ is a smooth function. Then, the spectra are shifted, but there is no essential difference. Therefore, let us assume the simplest form, Eq.(1). 
$\lambda_{1} \leq \lambda_{2} \cdots \uparrow \infty \equiv\left\{\lambda_{k}\right\}$. If $f$ is expanded in terms of the eigenfunctions $\left\{\phi_{k}\right\}$, $f(\cdot)=\sum_{k=0}^{\infty} \alpha_{k} \phi_{k}(\cdot)$, then, $\sigma_{\mathcal{G}}[f]=\frac{1}{2} \sum_{k=0}^{\infty} \lambda_{k} \phi_{k}^{2}$. By choosing $F(x)=\exp -x$, thus, $P_{\mathcal{G}}[f]=\exp -\frac{1}{2} \sum_{k=0}^{\infty} \lambda_{k} \phi_{k}^{2}$. This satisfies (I) obviously by choosing a suitable measure for the functional integral. Noting that the smaller (larger) $\lambda_{k}$ in $\left\{\lambda_{k}\right\}$ coarsely reflects the larger (smaller) scale behavior of $\mathcal{G}$, the above choice also satisfies (II). (Compare two values of $P_{\mathcal{G}}[f]$ for, say, $f \sim \phi_{3}$ and $f \sim \phi_{100}$.)

Now, we shall formally generalize the procedure in the preliminary consideration. First, from the normalization requirement of $P_{\mathcal{G}}[\cdot]$ in (I), we find

$$
[d f]=\prod_{k=1}^{\infty} \sqrt{\frac{\lambda_{k}}{2 \pi}} d \alpha_{k}
$$

where the zero-mode $\lambda_{0}$ is understood to be removed if it appears (e.g. the case of $\partial \mathcal{M}=\emptyset)$. Formally repeating the same procedure as in the preliminary consideration, then,

$$
\begin{aligned}
\xi\left(\mathcal{G}, \mathcal{G}^{\prime}\right) & =\int[d f]\left(P_{\mathcal{G}}[f] P_{\mathcal{G}^{\prime}}[f]\right)^{\frac{1}{2}} \\
& =\int \prod_{k=1}^{\infty} \frac{d \alpha_{k}}{(2 \pi)^{\frac{1}{2}}}\left(\lambda_{k} \lambda^{\prime}{ }_{k}\right)^{\frac{1}{4}} \exp -\frac{1}{4} \Sigma_{m=1}^{\infty}\left(\lambda_{m}+\lambda^{\prime}{ }_{m}\right) \alpha_{m}^{2} \\
& =\prod_{k=1}^{\infty}\left\{\frac{1}{2}\left(\sqrt{\frac{\lambda_{k}}{\lambda_{k}^{\prime}}}+\sqrt{\frac{\lambda_{k}^{\prime}}{\lambda_{k}}}\right)\right\}^{-\frac{1}{2}}
\end{aligned}
$$

Thus,

$$
\begin{aligned}
d\left(\mathcal{G}, \mathcal{G}^{\prime}\right) & =-\ln \xi\left(\mathcal{G}, \mathcal{G}^{\prime}\right) \\
& =\frac{1}{2} \sum_{k=1}^{\infty} \ln \frac{1}{2}\left(\sqrt{\frac{\lambda_{k}}{\lambda_{k}^{\prime}}}+\sqrt{\frac{\lambda_{k}^{\prime}}{\lambda_{k}}}\right) .
\end{aligned}
$$

For brevity, let us refer to this quantity as the 'spectral distance' between $\mathcal{G}$ and $\mathcal{G}^{\prime}$. 
Finally, let us note one subtlety included in the above procedure for deriving Eq. (2): In taking the overlapping functional integral, $\int[d f]\left(P_{\mathcal{G}}[f] P_{\mathcal{G}^{\prime}}[f]\right)^{\frac{1}{2}}$, a prescription for the identification of a function $f$ defined on $\mathcal{G}$ with another function $f$ defined on $\mathcal{G}^{\prime}$ should be fixed. Here, we have chosen the following prescription: Let $\mathcal{G}$ and $\mathcal{G}^{\prime}$ be compact Riemannian manifolds, and $D$ be an elliptic differential operator. Let $0=\lambda_{0}<\lambda_{1} \leq \lambda_{2} \cdots \uparrow \infty \equiv\left\{\lambda_{k}\right\}$ and $0=\lambda_{0}^{\prime}<\lambda_{1}^{\prime} \leq \lambda_{2}^{\prime} \cdots \uparrow \infty \equiv\left\{\lambda_{k}^{\prime}\right\}$ are spectra of $D$ on $\mathcal{G}$ and $\mathcal{G}^{\prime}$, respectively, numbered in the increasing order. Then, we have sets of eigenfunctions of $D,\left\{\phi_{k}(\cdot)\right\}$ and $\left\{\phi_{k}^{\prime}(\cdot)\right\}$, corresponding to $\left\{\lambda_{k}\right\}$ and $\left\{\lambda_{k}^{\prime}\right\}$, respectively. A function $f(\cdot)$ on $\mathcal{G}$ can be expanded in terms of $\left\{\phi_{k}(\cdot)\right\}$, $f(\cdot)=\sum_{k} \alpha_{k} \phi_{k}(\cdot)$. Then, our prescription is that $f(\cdot)$ on $\mathcal{G}$ is identified w.r.t. $D$ with a function $\sum_{k} \alpha_{k} \phi_{k}^{\prime}(\cdot)$ on $\mathcal{G}^{\prime}$.

In cases when $\mathcal{G}$ and $\mathcal{G}^{\prime}$ are within some one-parameter family of geometries, this prescription may be justified rigorously through the adiabatic theorem [21]. Therefore, this way of identification seems to be the most natural one. This subtlety of identifying functions on different spaces also emerges in quantum field theory on a curved spacetime. The same prescription is implicitly adopted in this case (See the next subsection for more details). Even if there is a better prescription, the basic idea and procedures remain same, and only the final result Eq.(2) would be subject to some modification. Here, let us adopt the above-mentioned prescription.

\subsection{The Physical INTERPREtation of the SPECtral Distance}

Physically, it is natural to expect that the geometrical information reflects on the behavior of a field distributed on $(\mathcal{M}, g)$. In fact, the functional $\sigma[\cdot]$ (Eq.(1)) is in the form of the action of a scalar field on $(\mathcal{M}, g)$. (Note, however, that $(\mathcal{M}, g)$ is Riemannian.) Thus, one may suppose that the spectral distance $d\left(\mathcal{G}, \mathcal{G}^{\prime}\right)$ yields a physical interpretation. Indeed, the quantity $\xi\left(\mathcal{G}, \mathcal{G}^{\prime}\right)=\exp -d\left(\mathcal{G}, \mathcal{G}^{\prime}\right)$ (Eqs. $(2)$ and (3)) turns out to be related to a reduced density matrix element for the universe, which appears in the discussions of the emergence of the classical world from the quantum universe [22],[23],[24]. Let us now see this point in order to make clear the 
assumptions behind this coincidence, i.e. the relation of $d\left(\mathcal{G}, \mathcal{G}^{\prime}\right)$ with a reduced density matrix element.

Let us consider the system of gravity and a massless scalar field. It is interesting to consider other kinds of fields also, the Dirac field for instance. However, the structure of the vacuum state for a fermion field looks quite different from the one for a boson field, at least mathematically, and requires separate investigations. To avoid extra complications, therefore, let us consider only a scalar field here (Assumption a). The total action is given by (the signature is chosen as $(-,+, \cdots,+))$

$$
\begin{aligned}
S[\mathcal{G}, \phi] & =1 / \alpha \cdot \int R \sqrt{-g}+\int\left(-\frac{1}{2} \partial_{a} \phi \partial^{a} \phi-\frac{1}{2} m^{2} \phi^{2}\right) \sqrt{-g} \\
& =: S_{\text {grav }}[\mathcal{G}]+S_{\text {matter }}[\mathcal{G}, \phi]
\end{aligned}
$$

where $\alpha$ is a suitable gravitational constant. Here, the spatial geometry $\mathcal{G}$ and the scalar field $\phi$ induced on $\mathcal{G}$ are the configuration variables. This system obeys the quantum theory, described by the Wheeler-DeWitt equation $[18], H \Psi[\mathcal{G}, \phi]=0$, where $H$ is the Hamiltonian constraint obtained from $S[\mathcal{G}, \phi]$. In the semiclassical region, it may be a good approximation to do the quantum theory separately for $S_{\text {grav }}(\mathcal{G})$ and $S_{\text {matter }}[\mathcal{G}, \phi]:$ On the one hand, we regard that the dynamics of $\mathcal{G}$ is approximately described solely by $S_{\text {grav }}[\mathcal{G}]$; On the other hand, $\phi$ is described by $S_{\text {matter }}[\mathcal{G}, \phi]$, with $\mathcal{G}$ treated just as parameters $(\text { Assumption } b)^{\star}$. Thus we set the Ansatz for $\Psi(\mathcal{G}, \phi)$ as, $\Psi(\mathcal{G}, \phi)=\varphi(\mathcal{G}) \cdot \eta(\mathcal{G}, \phi)$.

Now, let us consider $\eta[\mathcal{G}, \phi]$, described by $S_{\text {matter }}[\mathcal{G}, \phi]$. Choosing $N=1, N_{i}=$

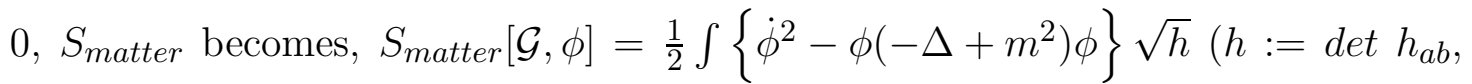
$h_{a b}$ is the induced spatial metric). It is natural to expand $\phi(t, \vec{x})$ in terms of the normalized eigenfunctions, $\left\{\phi_{k}(\vec{x})\right\}$, of the elliptic operator $D:=-\Delta+m^{2}$, satisfying eigenvalue equations $D \phi_{k}(\cdot)=\lambda_{k}(\mathcal{G}) \phi_{k}(\cdot)$, with a suitable boundary

* There is no essential difference between the massless case and the massive case.

* Usually, this treatment is regarded as valid on grounds of the 'smallness' of $\alpha$. More rigorously, the typical amplitude of quantum fluctuations of spacetime should be taken into considerations for the justification of this treatment [4]. 
condition compatible with the action principle for $S_{\text {matter }}[\mathcal{G}, \phi]$. Thus, $\phi(t, \cdot)=$ $\sum_{k} \alpha_{k}(t) \phi_{k}(\cdot)$. Then, $S_{\text {matter }}[\mathcal{G}, \phi]=\sum_{k} \frac{1}{2} \int\left(\dot{\alpha}_{k}^{2}-\lambda_{k}(\mathcal{G}) \alpha_{k}^{2}\right) d t$, which is equivalent to a collection of harmonic oscillators (note that $\mathcal{G}$ is now treated as a fixed parameter). Afterwards, it is in principle straightforward to quantize this system. Technically, the simplest way to quantize it is to choose the adiabatic vacuum as quantum state (Assumption c): We assume that the typical time scale of the change in the field $\phi$ is much shorter than the one of the change in geometry $\mathcal{G}$. Then we define the vacuum state at each instant of time;

$$
\eta(\mathcal{G}, \phi)=\prod_{k}\left(\frac{\lambda_{k}(\mathcal{G})}{2 \pi}\right)^{1 / 4} \exp -\frac{1}{4} \lambda_{k}(\mathcal{G}) \alpha_{k}^{2}
$$

The density matrix of the universe may be defined as

$$
\rho\left(\mathcal{G}, \phi ; \mathcal{G}^{\prime}, \phi^{\prime}\right)=\Psi(\mathcal{G}, \phi) \Psi^{*}\left(\mathcal{G}^{\prime}, \phi^{\prime}\right)
$$

To give the meaning to this density matrix, the wavefunction $\Psi(\mathcal{G}, \phi)$ should yield the probabilistic interpretation. It means that $\Psi(\mathcal{G}, \phi)$ should be understood as normalized w.r.t. a suitable inner product, or some alternative interpretation for $\Psi(\mathcal{G}, \phi)$ should be provided (Assumption $d$ ). To discuss the quantum decoherence of the universe, one may treat $\mathcal{G}$ as the system variable and $\phi$ as the environment, and take the partial trace w.r.t. $\phi$ (remember the Ansatz for $\Psi(\mathcal{G}, \phi)$ ):

$$
\begin{aligned}
\rho_{\text {reduced }}\left(\mathcal{G}, \mathcal{G}^{\prime}\right) & =\int[d \phi] \rho\left(\mathcal{G}, \phi ; \mathcal{G}^{\prime}, \phi\right)=\varphi(\mathcal{G}) \varphi^{*}\left(\mathcal{G}^{\prime}\right) \int[d \phi] \eta(\mathcal{G}, \phi) \eta^{*}\left(\mathcal{G}^{\prime}, \phi\right) \\
& =: \varphi(\mathcal{G}) \varphi^{*}\left(\mathcal{G}^{\prime}\right) \xi\left(\mathcal{G}, \mathcal{G}^{\prime}\right)
\end{aligned}
$$

where $\xi\left(\mathcal{G}, \mathcal{G}^{\prime}\right)$ exactly agrees with the one given by Eq.(2), the latter being expressed as $\xi\left(\mathcal{G}, \mathcal{G}^{\prime}\right)=\exp -d\left(\mathcal{G}, \mathcal{G}^{\prime}\right)$. Therefore, in our terms, the longer the

$\S$ It is notable that $\phi$ appears in the theory only through a set of expansion coefficients $\left\{\alpha_{k}\right\}$ w.r.t $\left\{\phi_{k}(\cdot)\right\}$, a set of eigenfunctions for $D$. In this sense, $\left\{\alpha_{k}\right\}$ can be regarded more fundamental than $\phi$ itself. 
spectral distance $d\left(\mathcal{G}, \mathcal{G}^{\prime}\right)$ is, the smaller the corresponding off-diagonal element $\rho_{\text {reduced }}\left(\mathcal{G}, \mathcal{G}^{\prime}\right)$ is, implying the stronger decoherence between the universes $\mathcal{G}$ and $\mathcal{G}^{\prime}$.

In taking the partial trace w.r.t. $\phi$, the operation $\int[d \phi] \eta(\mathcal{G}, \phi) \eta^{*}\left[\mathcal{G}^{\prime}, \phi\right]$ should be of meaning. This procedure contains two subtle points: First, it corresponds to comparing states for two different harmonic oscillators with different frequencies $\left(\lambda_{k}(\mathcal{G})\right.$ and $\left.\lambda_{k}\left(\mathcal{G}^{\prime}\right)\right)$. Mathematically, it is just a change of basis in a functional space. However, physically it presupposes the identification of two Hilbert spaces, one characterized by $\lambda_{k}(\mathcal{G})$, and the other by $\lambda_{k}\left(\mathcal{G}^{\prime}\right)$. This is not within the framework of ordinary quantum theory. Secondly, a reasonable prescription to identify a function $\phi$ on $\mathcal{G}$ with the same on $\mathcal{G}^{\prime}$ should be fixed. The rule adopted here is to identify $\sum_{k} \alpha_{k} \phi_{k}(\cdot)$ with $\sum_{k} \alpha_{k} \phi^{\prime}{ }_{k}(\cdot)$. Here, $\left\{\phi_{k}(\cdot)\right\}$ and $\left\{\phi^{\prime}{ }_{k}(\cdot)\right\}$ are eigenfunctions of $D$ on $\mathcal{G}$ and $\mathcal{G}^{\prime}$, respectively. Thus, we face with the same subtlety of identifying functions defined on $\mathcal{G}$ and $\mathcal{G}^{\prime}$ as discussed in $\S \S 2-1$. These subtleties always emerge, explicitly or implicitly, whenever we discuss the quantum field theory in curved spacetime. All we can do at present is to expect that once the inner product in a space of wavefunctions of the universe, $\Psi(\mathcal{G}, \phi)$, shall be correctly fixed, and a complete interpretation of $\Psi(\mathcal{G}, \phi)$ shall be given, this problem would be automatically solved (Assumption e). (Therefore, Assumptions $d$ and $e$ are deeply linked with each other.)

Ref.[22] may be one of the earliest works which pays attention to the reduced density matrix for the explanation of the classical behavior of the quantum universe. Ref.[23] pursues this idea explicitly for the case of the perturbed Friedmann universe with a massive scalar field, based on the model of Ref.[25]. In this case, unperturbed quantities, i.e. the scale factor $a(t)$ and the homogeneous component of the scalar field, $\phi(t)$, correspond to our $\mathcal{G}$. For the tensor modes of pertur-

* One situation in usual quantum theory, which is of some similarity with the present problem, appears in the discussions of the adiabatic perturbations [21]. In this case, the Hilbert space for the system characterized by parameters, say $\omega$, can be asymptotically related with the other Hilbert space characterized by $\omega^{\prime}$, when $\omega \rightarrow \omega^{\prime}$. However, the problem of our concern requires even the comparison of two drastically different Hilbert spaces. 
bations, for instance, one can essentially set $\lambda_{k}=\frac{k}{2} a^{2}$. Then the suppression factor $\xi\left(a, a^{\prime}\right)=\lim _{N \rightarrow \infty} \exp -\frac{N}{4 a a^{\prime}}\left(a-a^{\prime}\right)^{2}$ appears, which agrees with Eq.(2), with $\lambda_{k}=\frac{k}{2} a^{2}{ }^{\star}$. Ref.[24] recapitulates the arguments of Ref.[22] and [23] in more general terms. It even implies the idea of the distance, though no further investigations (e.g. its convergence condition, comparison with the axioms of distance) are pursued. Needless to say, these works, [22-24], are all subject to the assumptions and subtleties pointed out above.

Let us summarize the physical interpretation of the spectral distance $d\left(\mathcal{G}, \mathcal{G}^{\prime}\right)$ introduced in $\S \S 2-1:$ The quantity $\xi\left(\mathcal{G}, \mathcal{G}^{\prime}\right)=\exp -d\left(\mathcal{G}, \mathcal{G}^{\prime}\right)$ boils down to a reduced density matrix element (for a system of the universe $(\mathcal{G})$ and a scalar field $(\phi)$, with $\phi$ being traced out) under the following conditions:

(a) A suitable inner product between the wavefunctions of the universe, $\Psi(\mathcal{G}, \phi)$ and $\Psi\left(\mathcal{G}^{\prime}, \phi^{\prime}\right)$ can be defined, so that the density matrix admits quantum theoretical interpretation.

(b) The spacetime can be treated semiclassically, and the scalar field $\phi$ on $\mathcal{G}$ and $\mathcal{G}^{\prime}$ is in the ground state and can be treated adiabatically (the time scale of the change in the field is regarded as much shorter than the one of the change in geometry).

This coincidence of $\xi\left(\mathcal{G}, \mathcal{G}^{\prime}\right)$ boiling down to the reduced density-matrix element suggests several important things:

First, the specific choice of $F(x)=\exp -x$ in $§ \S 2-1$ is clearly distinguished amongst several possibilities, from the view of physical applications.

Secondly, once we obtain a satisfactory quantum theory of gravity in the future to compute $\rho_{\text {reduced }}\left(\mathcal{G}, \mathcal{G}^{\prime}\right)$ exactly, it would be relevant to define 'closeness' between $\mathcal{G}$ and $\mathcal{G}^{\prime}$ as $-\ln \left\{\rho_{\text {reduced }}\left(\mathcal{G}, \mathcal{G}^{\prime}\right) / \varphi(\mathcal{G}) \varphi^{*}\left(\mathcal{G}^{\prime}\right)\right\}$. In other words, the stronger two

$\star$ More precisely, because $\phi(t)$ is also included in the category of our $\mathcal{G}$, the combination $\frac{k}{2} a^{2}-i 2 a^{3} m \phi$ is to be identified with $\lambda_{k}$, yielding the factor

$\xi\left(a, \phi ; a^{\prime}, \phi^{\prime}\right)=\lim _{N \rightarrow \infty} \exp -\frac{N}{4 a a^{\prime}}\left(a-a^{\prime}\right)^{2} \exp -\frac{\pi^{2}}{4} m^{2} a a^{\prime}\left(\phi-\phi^{\prime}\right)^{2}[23]$. 
geometries interfere with each other quantum mechanically, the 'closer' they can be regarded (a suggestion from physics for mathematics).

Thirdly, one may be able to estimate $\rho_{\text {reduced }}\left(\mathcal{G}, \mathcal{G}^{\prime}\right) / \varphi(\mathcal{G}) \varphi^{*}\left(\mathcal{G}^{\prime}\right)$ by calculating $\exp -d\left(\mathcal{G}, \mathcal{G}^{\prime}\right)$ according to (3) (a suggestion from mathematics for physics). Needless to say, we have no satisfactory theory of quantum gravity at present, so that $\rho_{\text {reduced }}\left(\mathcal{G}, \mathcal{G}^{\prime}\right)$ cannot be calculated exactly. However, the reduction of $d\left(\mathcal{G}, \mathcal{G}^{\prime}\right)$ (which has been derived from general arguments and which is applicable to any geometries in principle) into $-\ln \left\{\rho_{\text {reduced }}\left(\mathcal{G}, \mathcal{G}^{\prime}\right) / \varphi(\mathcal{G}) \varphi^{*}\left(\mathcal{G}^{\prime}\right)\right\}$ (estimated approximately) when $\mathcal{G}$ and $\mathcal{G}^{\prime}$ are restricted to be of the same dimensionality and topology, is very suggestive and should be meaningful. Thus, we can infer the following equality,

$$
d\left(\mathcal{G}, \mathcal{G}^{\prime}\right)=-\ln \left\{\rho_{\text {reduced }}\left(\mathcal{G}, \mathcal{G}^{\prime}\right) / \varphi(\mathcal{G}) \varphi^{*}\left(\mathcal{G}^{\prime}\right)\right\}
$$

In connection with the condition $(a)$, we should note the following: The inner product between two wavefunctions defined on two different superspaces is much less known than the inner product between wavefunctions defined on the same superspace. (For instance, universes with different dimensions, or with different global topologies are subject to different superspaces.) In other words, there is no established way of comparing universes with different global structures. This is the main obstacle which prevents us from undertaking extensive studies on the phenomenon of the topology change. At the same time, this is one of the motivations of the introduction of the spectral distance $(\S 1)$. At present, we are usually forced to restrict ourselves to the comparably moderate cases in which universes lie in the same superspace (in most cases, minisuperspace or its generalization), assuming somehow the inner product.

Regarding the relation between the spectral distance and the reduced density matrix, therefore, one had better keep the following caveat in mind: Only for the cases when $\mathcal{G}$ and $\mathcal{G}^{\prime}$ are subject to the same superspace (typically, of the same dimension and topology), the above equality holds safely under several assumptions. For other cases, it should be regarded as an extrapolation. 
Since this caveat should be always remembered whenever we shall connect the spectral distance with physical interpretations, we refer to it as Caveat $A$, for brevity. It is quite probable that (some modified form of) the above equality turns out to be generally true, once a satisfactory theory of quantum gravity is provided.

\subsection{The CONVERGence CONDition of the SPeCtral Distance}

Let us now investigate the necessary condition for the convergence of the spectral distance, since its definition in Eq.(3) includes an infinite summation. Clearly, the necessary condition for convergence is

$$
a_{k}\left(\mathcal{G}, \mathcal{G}^{\prime}\right):=\frac{1}{2}\left(\sqrt{\frac{\lambda_{k}}{\lambda^{\prime}{ }_{k}}}+\sqrt{\frac{\lambda^{\prime}}{\lambda_{k}}}\right) \longrightarrow 1 \text { as } k \rightarrow \infty
$$

Note that $a_{k} \geq 1,=\Longleftrightarrow \lambda_{k}=\lambda^{\prime}{ }_{k}$. Thus the necessary condition for the convergence of $d\left(\mathcal{G}, \mathcal{G}^{\prime}\right)$ is $\frac{\lambda_{k}^{\prime}}{\lambda_{k}} \rightarrow 1$ as $k \rightarrow \infty$. Now, we have Weyl's asymptotic formula [26],[27], which can be represented in several ways,

$$
\begin{aligned}
\sum_{k=0}^{\infty} \exp -\lambda_{k} t & =\frac{1}{(4 \pi t)^{\frac{n}{2}}} V+O(1) \text { as } t \downarrow 0, \\
N(\Lambda) & \sim \frac{\omega_{n} V}{(2 \pi)^{n}} \Lambda^{\frac{n}{2}} \text { as } \Lambda \rightarrow \infty, \\
\lambda_{k} & \sim\left(\frac{(2 \pi)^{n}}{\omega_{n} V} k\right)^{\frac{2}{n}} \text { as } k \rightarrow \infty,
\end{aligned}
$$

where $n=\operatorname{dim} \mathcal{M}=$ dimension of $\mathcal{M}, V=\operatorname{vol} \mathcal{M}=n$-volume of $\mathcal{M}, N(\Lambda):=$ $\#\left\{\lambda_{k} \mid \lambda_{k} \leq \Lambda\right\}$ and $\omega_{n}:=n$-volume of unit $n$-disk in $\mathbf{R}^{n}$ (e.g. $\left.\omega_{2}=\pi\right)$. Significantly, the ' $O(1)$-term' in $(4-a)$ reduces to $\chi(\mathcal{M}) / 6+O(t)$ when $n=2, \mathcal{M}$ is compact, and $\partial \mathcal{M}=\emptyset$, where $\chi(\mathcal{M})$ is the Euler number of $\mathcal{M}$ [26]. We shall come across the application of this result in $\S 3$. Weyl's asymptotic formula $(4-c)$ states that the asymptotic behavior of $\lambda_{k}(k \rightarrow \infty)$ depends on $\operatorname{dim} \mathcal{M}$, volM $\mathcal{M}$, and topology of $\mathcal{M}$, but does not depend on a detailed local geometry of $(\mathcal{M}, g)$. From 
$(4-c)$

$$
\frac{\lambda_{k}^{\prime}}{\lambda_{k}} \sim \frac{\left(\omega_{n} V\right)^{\frac{2}{n}}}{\left(\omega_{n^{\prime}} V^{\prime}\right)^{\frac{2}{n^{\prime}}}} k^{\frac{2}{n^{\prime}}-\frac{2}{n}} \text { as } k \rightarrow \infty
$$

Thus the necessary condition for convergence of $d\left(\mathcal{G}, \mathcal{G}^{\prime}\right)$ is

$$
\operatorname{dim} \mathcal{M}=\operatorname{dim} \mathcal{M}^{\prime} \text { and } \operatorname{vol} \mathcal{M}=\operatorname{vol} \mathcal{M}^{\prime}
$$

This result is quite suggestive. According to the density-matrix interpretation with Caveat $A$, this suggests that two universes with different dimension or volume decohere very strongly.

Finally, we should note that Eq.(5) is just the necessary condition for convergence, but not a sufficient one.

\subsection{The SCALE-DEPENDENT SPECTRAL DistanCE}

From the formula Eq.(3), we are naturally led to the scale-dependent spectral distance, by introducing a cut-off in the summation. We shall compare the subsets of $\left\{\lambda_{k}\right\}$ and $\left\{\lambda_{k}^{\prime}\right\}$ constructed from elements less than $\Lambda$. It corresponds to a coarse comparison of two geometries $\mathcal{G}$ and $\mathcal{G}^{\prime}$, neglecting the difference in the smaller scale behaviors than $\Lambda^{-\frac{1}{2}}$.

More specifically, let us define

$$
N_{\Lambda}:=\operatorname{Min}\left(\#\left\{\lambda_{k} \in\left\{\lambda_{k}\right\} \mid 0 \leq \lambda_{k} \leq \Lambda\right\}, \quad \#\left\{\lambda_{k}^{\prime} \in\left\{\lambda_{k}^{\prime}\right\} \mid 0 \leq \lambda_{k}^{\prime} \leq \Lambda\right\}\right)
$$

In terms of $N_{\Lambda}$, we shall define $\Lambda_{k}^{\Lambda}$ as,

$$
\lambda_{k}^{\Lambda}:= \begin{cases}\lambda_{k} & \text { for } k \leq N_{\Lambda} \\ \lambda_{N_{\Lambda}} & \text { for } k>N_{\Lambda}\end{cases}
$$

then, $\left\{\lambda_{k}^{\Lambda}\right\}=\left\{\lambda_{0}, \lambda_{1}, \cdots, \lambda_{N_{\Lambda}}, \lambda_{N_{\Lambda}}, \lambda_{N_{\Lambda}}, \cdots\right\}$. In the same way, we shall define $\left\{\lambda_{k}^{\prime \Lambda}\right\}=\left\{\lambda_{0}^{\prime}, \lambda_{1}^{\prime}, \cdots, \lambda_{N_{\Lambda}}^{\prime}, \lambda_{N_{\Lambda}}^{\prime}, \lambda_{N_{\Lambda}}^{\prime}, \cdots\right\}$. Then, we shall define the scale-dependent 
spectral distance $d_{\Lambda}\left(\mathcal{G}, \mathcal{G}^{\prime}\right)$ as

$$
d_{\Lambda}\left(\mathcal{G}, \mathcal{G}^{\prime}\right)=\frac{1}{2} \Sigma_{k=1}^{N_{\Lambda}} \ln \frac{1}{2}\left(\sqrt{\frac{\lambda_{k}^{\Lambda}}{\lambda_{k}^{\prime \Lambda}}}+\sqrt{\frac{\lambda_{k}^{\prime \Lambda}}{\lambda_{k}^{\Lambda}}}\right)
$$

There are other possibilities in the way of introducing a cut-off. For instance, instead of taking the minimum of the two numbers in the definition of $N_{\Lambda}$, taking the average of the two numbers is one possibility. Replacing minimum by maximum is another possibility. Weyl's asymptotic formula Eq. $(4-c)$ guarantees that the difference caused by different choices becomes negligible when $\Lambda$ is sufficiently large.

It is interesting to investigate the behavior $d_{\Lambda}\left(\mathcal{G}, \mathcal{G}^{\prime}\right)$ as a function of $\Lambda$ for given $\mathcal{G}$ and $\mathcal{G}^{\prime}$. For instance, suppose a geometry $\mathcal{G}$ with a very complicated topological structure, $\mathcal{G}=\mathcal{G}^{\prime} \# h_{1} \# h_{2} \cdots \# h_{m}$, where $h_{i}$ 's are some topological handles of a typical scale $l$. When the cut-off parameter $\Lambda$ is increased smoothly, the spectral distance $d_{\Lambda}\left(\mathcal{G}, \mathcal{G}^{\prime}\right)$ is expected to increase abruptly near $\Lambda \sim l^{-2}$, indicating that $\mathcal{G}$ and $\mathcal{G}^{\prime}$ are almost similar in the scale larger than $l$, but they are very different in the scale smaller than $l$. This provides a new quantitative representation of the scaledependent topology or topological approximation [10]. As already mentioned in $\S 1$, the concept of scale-dependent topology or topological approximation has many interesting applications. However, its rigorous quantitative formulation is quite difficult: It requires the concept of 'closeness' between two topologically different Riemannian manifolds [10], for which we have no mathematical theory as yet. To make this concept of 'closeness' or 'distance' physically sensible, it should have some connection with physical quantities. In this respect, the quantity $d_{\Lambda}\left(\mathcal{G}, \mathcal{G}^{\prime}\right)$ is a good candidate for the measure of closeness between $\mathcal{G}$ and $\mathcal{G}^{\prime}$, since it is defined in terms of spectra (where a matter field plays the role of a probe for the geometrical structure of the universe) and since it can be related to the reduced density-matrix element for the universe (with Caveat $A$ ) as discussed in §§2-2. In Ref.[10], the quantitative description of scale-dependent topology has been investigated in terms of the scattering cross-sections, treating topological handles as a scatterer. One 
restriction of this framework is that it requires an asymptotic region with trivial topology to set up a scattering problem. On the other hand, the description in terms of the scale-dependent spectral distance does not assume such an asymptotic region.

\subsection{The FAILURE OF THE TRIANGUlar INEQUALITY}

The ordinary requirements for a function $d: S \times S \rightarrow \mathbf{R}(S:$ a set) to be regarded as a distance are

(I) Positivity: $(a) d(p, q) \geq 0 \quad(b) d(p, q)=0 \Longleftrightarrow p=q$

(II) Symmetry: $d(p, q)=d(q, p)$.

(III) The triangular inequality: $d(p, q)+d(q, r) \geq d(p, r)$.

The spectral distance $d\left(\mathcal{G}, \mathcal{G}^{\prime}\right)$ clearly satisfies $(I)-(a)$ and $(I I)$. As for $(I)-(b)$, we know counter-examples which do not satisfy ' $\Rightarrow$ ': There are examples of a pair of non-isometric geometries $\mathcal{G}$ and $\mathcal{G}^{\prime}$ whose spectra are identical $\left\{\lambda_{k}\right\} \equiv\left\{\lambda^{\prime}{ }_{k}\right\}$. Two non-isometric geometries on $T^{16}$ with identical spectra have been given by Milnor [14]. Later, other examples have also been constructed [15],[16] and they are called isospectral manifolds [17]. (Note that the necessary conditions for $\mathcal{G}$ and $\mathcal{G}^{\prime}$ to be isospectral, are $\operatorname{dim} \mathcal{M}=\operatorname{dim} \mathcal{M}^{\prime}$ and $\operatorname{vol} \mathcal{M}=\operatorname{vol} \mathcal{M}^{\prime}$ by Weyl's asymptotic formula Eq. $(4-c)$.) Such a pair of isospectral manifolds cannot be separated in terms of the spectral distance w.r.t. the Laplacian $\Delta^{\star}$. These isospectral manifolds do not seem to be generic, so that they may be identified in the space of all geometries. On the identified space, $(I)-(b)$ can be regarded to hold good. This observation implies that the spectral distance is a 'coarser' distance compared with the distance defined by the DeWitt metric [18] (for manifolds with the same dimension, volume and topology). Employing the density-matrix interpretation of the spectral distance, it might imply that a pair of isospectral spaces interfere with

\footnotetext{
* However, some other elliptic operators can yield the spectral distance which distinguishes the isospectral manifolds w.r.t. $\Delta$.
} 
each other strongly. However, it is uncertain as to what extent these exceptional cases have an influence on the applications to cosmology.

Finally, we investigate the validity of $(I I I)$ the triangular inequality, $d\left(\mathcal{G}, \mathcal{G}^{\prime}\right)+$ $d\left(\mathcal{G}^{\prime}, \mathcal{G}^{\prime \prime}\right) \geq d\left(\mathcal{G}, \mathcal{G}^{\prime \prime}\right)$. We mention in advance that in $\S 3$, where 2-dimensional models shall be investigated, many cases shall be found in which the triangular inequality does not hold. Thus, the spectral distance does not satisfy the triangular inequality in general. The spectral distance can be written as $d\left(\mathcal{G}, \mathcal{G}^{\prime}\right)=$ $\frac{1}{2} \sum_{k=1}^{\infty} d_{k}\left(\mathcal{G}, \mathcal{G}^{\prime}\right)$ (see Eq.(3)). It is interesting to investigate the condition for the term-wise violation of the triangular inequality: First,

$$
d_{k}\left(\mathcal{G}, \mathcal{G}^{\prime}\right)+d_{k}\left(\mathcal{G}^{\prime}, \mathcal{G}^{\prime \prime}\right)=\ln \frac{1}{2}\left\{\frac{1}{2}\left(1+\frac{1}{\alpha}\right) \sqrt{\beta}+\frac{1}{2}(1+\alpha) \frac{1}{\sqrt{\beta}}\right\}
$$

where $\alpha:=\sqrt{\frac{\lambda_{k}}{\lambda^{\prime}{ }_{k}}}$ and $\beta:=\sqrt{\frac{\lambda_{k}}{{\lambda^{\prime \prime}}_{k}}}$. Next,

$$
d_{k}\left(\mathcal{G}, \mathcal{G}^{\prime \prime}\right)=\ln \frac{1}{2}\left(\sqrt{\beta}+\frac{1}{\sqrt{\beta}}\right)
$$

Now, $\frac{1}{2}\left(1+\alpha^{-1}\right) \sqrt{\beta}+\frac{1}{2}(1+\alpha) \sqrt{\beta}^{-1}-\left(\sqrt{\beta}+\sqrt{\beta}^{-1}\right)=-(1-\alpha)\{2 \alpha \sqrt{\beta}(\alpha-\beta)\}^{-1}$, which is negative when $\beta<\alpha<1$ or $\beta>\alpha>1$. Thus,

$$
d_{k}\left(\mathcal{G}, \mathcal{G}^{\prime}\right)+d_{k}\left(\mathcal{G}^{\prime}, \mathcal{G}^{\prime \prime}\right)<d_{k}\left(\mathcal{G}, \mathcal{G}^{\prime \prime}\right) \Longleftrightarrow \lambda_{k}<\lambda^{\prime}{ }_{k}<\lambda^{\prime \prime}{ }_{k} \text { or } \lambda_{k}>\lambda^{\prime}{ }_{k}>\lambda^{\prime \prime}{ }_{k}
$$

Three geometries $\mathcal{G}, \mathcal{G}^{\prime}$ and $\mathcal{G}^{\prime \prime}$ satisfying $\lambda_{k}<\lambda^{\prime}{ }_{k}<\lambda^{\prime \prime}{ }_{k}(k=1,2, \cdots)$ can easily be constructed if the difference in volumes is allowed. Note that the eigenvalues scale as $\lambda_{k} \propto V^{-\frac{2}{n}}$ w.r.t. $n$-volume $V$. Thus, any conformally equivalent geometries $\mathcal{G}, \mathcal{G}^{\prime}$ and $\mathcal{G}^{\prime \prime}$ such that vol $\mathcal{G}>$ vol $\mathcal{G}^{\prime}>$ vol $\mathcal{G}^{\prime \prime}$ satisfy this condition. Thus, $d_{\Lambda}\left(\mathcal{G}, \mathcal{G}^{\prime}\right)+d_{\Lambda}\left(\mathcal{G}^{\prime}, \mathcal{G}^{\prime \prime}\right)<d_{\Lambda}\left(\mathcal{G}, \mathcal{G}^{\prime \prime}\right)$ in this case. (The cut-off $\Lambda$ is needed, since volumes are different.) However, we also want to know the case of $d(\cdot, \cdot)$ (corresponding to the case of $\Lambda \rightarrow \infty$ ). We are then led to a question: Whether there exist three geometries $\mathcal{G}, \mathcal{G}^{\prime}$ and $\mathcal{G}^{\prime \prime}$ such that $\operatorname{dim} \mathcal{G}=\operatorname{dim} \mathcal{G}^{\prime}=\operatorname{dim} \mathcal{G}^{\prime \prime}$, $\operatorname{vol} \mathcal{G}=\operatorname{vol} \mathcal{G}^{\prime}=\operatorname{vol} \mathcal{G}^{\prime \prime}$ and $\lambda_{k}<\lambda^{\prime}{ }_{k}<\lambda^{\prime \prime}{ }_{k}(k=1,2, \cdots)$. 
This is a highly non-trivial question and it seems that the answer is not known as yet.

In this connection, let us remember the distance in the superspace defined by the DeWitt metric [18]. Although the DeWitt metric is not the positive definite metric, the latter is induced on a surface of a constant volume in the superspace. On this surface, then, the distance can be defined using this positive definite metric. Obviously, the distance thus defined satisfies the triangular inequality as in ordinary Riemannian geometry. Thus, the failure of the triangular inequality for the spectral distance (examples of which shall be shown in the next section) explicitly demonstrates that the spectral distance and the distance defined by the DeWitt metric are not equivalent to each other.

\section{Closeness between the orientable and the non-orientable universes}

We apply the spectral distance to quantum cosmology: We ask a question as to whether universes with different topologies interfere quantum mechanically. A probable answer which one might give would be that they decohere with each other since they 'sound' differently, resulting in a long spectral distance. To investigate this problem, let us set up several concrete models in 2-dimension with various topologies, and investigate the spectral distances between them in detail. In particular, we shall concentrate on the cases of

(A) $T^{2}$ and $\mathbf{R} P^{2} \# \mathbf{R} P^{2}$ (Klein's bottle).

(B) $S^{2}$ and $\mathbf{R} P^{2}$.

Here, $T^{2}$ is a covering space of $\mathbf{R} P^{2} \# \mathbf{R} P^{2}[11]$, and the former is orientable while the latter is non-orientable. The relation between $S^{2}$ and $\mathbf{R} P^{2}$ is also the same. We shall construct models of $T^{2}$ and $\mathbf{R} P^{2} \# \mathbf{R} P^{2}$, both of which are locally flat. Then, we can focus on the effect of the difference of global topologies, or the difference of orientabilities, in this case. We shall also construct models of $S^{2}$ and 
$\mathbf{R} P^{2}$, both of which are homogeneous (constant curvature spaces). By making the antipodal identification on a 2 -sphere with radius $\sqrt{2} R$, a homogeneous $\mathbf{R} P^{2}$ with 2 -volume $4 \pi R^{2}$ can be constructed [11]. Therefore, the difference between $S^{2}$ and $\mathbf{R} P^{2}$ in this case includes a difference of local curvature as well as a difference of orientability. Thus, this is the simplest case in which local as well as global geometries are different.

As discussed in the previous section, the spectral distance can be interpreted as $d\left(\mathcal{G}, \mathcal{G}^{\prime}\right)=-\ln \left\{\rho_{\text {reduced }}\left(\mathcal{G}, \mathcal{G}^{\prime}\right) / \varphi(\mathcal{G}) \varphi^{*}\left(\mathcal{G}^{\prime}\right)\right\}$, with Caveat $A$. Thus, the spectral distances for $(A)$ and $(B)$ provide us with some insights for our above-mentioned question.

\section{1. $T^{2}$ AND $\mathbf{R} P^{2} \# \mathbf{R} P^{2}$}

As the simplest class of models, we shall investigate spaces $(\Sigma)$ constructed as $\Sigma \simeq \mathbf{R}^{2} / G$, where $G$ is a discrete subgroup of the Euclid group of $\mathbf{R}^{2}$, acting freely on $\mathbf{R}^{2}$. There are only 5 types of spaces constructed in this manner; $\mathbf{R}^{2}$, a cylinder, a Möbius' strip, $T^{2}$ and $\mathbf{R} P^{2} \# \mathbf{R} P^{2}$ [28]. Amongst them, only $T^{2}$ and $\mathbf{R} P^{2} \# \mathbf{R} P^{2}$ are compact. Thus we choose these spaces as our models.

3.1.1 $T^{2} ;$ The case of $\tau^{1} \neq 0$.

A torus $T^{2}$ can be constructed by choosing as $G$, a translation group, $G=$ $\{m \vec{a}+n \vec{b}\}(m, n \in \mathbf{Z})$, where $\vec{a}=(1,0) / \sqrt{\tau^{2}}, \vec{b}=\left(\tau^{1}, \tau^{2}\right) / \sqrt{\tau^{2}}\left(\tau^{2}>0\right)$ and $\mathbf{Z}=\{0, \pm 1, \pm 2, \cdots\}$. Here a set of two real parameters $\left(\tau^{1}, \tau^{2}\right)\left(\tau^{2}>0\right)$ are the Teichmüller parameters, characterizing the global shape of a torus [29]. We fix the volume of $T^{2}$ to be unity, which is taken care of by the factor $\sqrt{\tau^{2}}$ in the choice of $\vec{a}$ and $\vec{b}$. It is convenient to introduce a coordinate $\left(\xi^{1}, \xi^{2}\right)$ defined by

$$
\left(\begin{array}{l}
x \\
y
\end{array}\right)=\frac{1}{\sqrt{\tau^{2}}}\left\{\xi^{1}\left(\begin{array}{l}
1 \\
0
\end{array}\right)+\xi^{2}\left(\begin{array}{l}
\tau^{1} \\
\tau^{2}
\end{array}\right)\right\} .
$$

\footnotetext{
$\star$ In this paper, $\tau^{2}$ always represents the second component of $\left(\tau^{1}, \tau^{2}\right)$, and not the square of $\tau:=\tau^{1}+i \tau^{2}$.
} 
In this coordinate system $\left(\xi^{1}, \xi^{2}\right), \vec{a}$ and $\vec{b}$ can be expressed as $\vec{a}=(1,0)$ and $\vec{b}=(0,1)$, and the identification by $G$ reads $\left(\xi^{1}, \xi^{2}\right) \sim\left(\xi^{1}+m, \xi^{2}+n\right)$ for $\forall m, \forall n \in \mathbf{Z}$.

The flat metric is given by $d s^{2}=d x^{2}+d y^{2}=h_{a b} d \xi^{1} d \xi^{2}$, where $h_{a b}=\frac{1}{\tau^{2}}\left(\begin{array}{cc}1 & \tau^{1} \\ \tau^{1} & |\tau|^{2}\end{array}\right)\left(|\tau|^{2}:=\left(\tau^{1}\right)^{2}+\left(\tau^{2}\right)^{2}\right)$. Thus, the Laplacian becomes

$$
\Delta=\frac{1}{\sqrt{h}} \partial_{a}\left(\sqrt{h} h^{a b} \partial_{b}\right)=\frac{1}{\tau^{2}}\left(|\tau|^{2} \partial_{1}^{2}-2 \tau^{1} \partial_{1} \partial_{2}+\partial_{2}^{2}\right)
$$

When $\tau^{1} \neq 0$, the spectra become,

$$
\lambda_{m n}=\frac{4 \pi^{2}}{\tau^{2}}\left(|\tau|^{2} m^{2}-2 \tau^{1} m n+n^{2}\right)=: 4 \pi^{2} Q(m, n)
$$

with normalized eigenfunctions

$$
\left\{\begin{array}{c}
u_{(0,0)}=1 \\
u_{(m, n)}=\sqrt{2} \cos \left(2 \pi m \xi^{1}+2 \pi n \xi^{2}\right) \\
v_{(m, n)}=\sqrt{2} \sin \left(2 \pi m \xi^{1}+2 \pi n \xi^{2}\right)
\end{array} \quad \text { for } \quad(m, n) \in \mathcal{R}-(0,0)\right.
$$

where $\mathcal{R}:=\mathbf{N}_{0} \times \mathbf{Z}-\{0\} \times(-\mathbf{N}), \mathbf{N}=\{1,2, \cdots\}$ and $\mathbf{N}_{0}:=\{0\} \cup \mathbf{N}$. It is convenient to represent the multiplicity of eigenvalues in the form of a 'spectral diagram' as shown in Figure $1-a$.

\subsection{2 $T^{2}$; The case of $\tau^{1}=0$.}

The case of $\tau^{1}=0$ should be treated separately. In this case, the Laplacian reduces to $\Delta=\frac{1}{\tau^{2}}\left(\left(\tau^{2}\right)^{2} \partial_{1}^{2}+\partial_{2}^{2}\right)$. The spectra become

$$
\lambda_{m n}=\frac{4 \pi^{2}}{\tau^{2}}\left(\left(\tau^{2}\right)^{2} m^{2}+n^{2}\right)=: 4 \pi^{2} Q_{0}(m, n)
$$


with normalized eigenfunctions

$$
\begin{aligned}
& u_{1(m, n)}= \begin{cases}1 & (m, n)=(0,0) \\
\sqrt{2} \cos 2 \pi m \xi^{1}, \sqrt{2} \cos 2 \pi n \xi^{2} & m, n \in \mathbf{N} \\
2 \cos 2 \pi m \xi^{1} \cos 2 \pi n \xi^{2} & (m, n) \in \mathbf{N} \times \mathbf{N}\end{cases} \\
& u_{2(m, n)}= \begin{cases}\sqrt{2} \sin 2 \pi n \xi^{2} & n \in \mathbf{N} \quad(m=0) \\
2 \cos 2 \pi m \xi^{1} \sin 2 \pi n \xi^{2} & (m, n) \in \mathbf{N} \times \mathbf{N}\end{cases} \\
& u_{3(m, n)}= \begin{cases}\sqrt{2} \sin 2 \pi m \xi^{1} & m \in \mathbf{N} \quad(n=0) \\
2 \sin 2 \pi m \xi^{1} \cos 2 \pi n \xi^{2} & (m, n) \in \mathbf{N} \times \mathbf{N}\end{cases} \\
& u_{4(m, n)}=2 \sin 2 \pi m \xi^{1} \sin 2 \pi n \xi^{2} \quad(m, n) \in \mathbf{N} \times \mathbf{N}
\end{aligned}
$$

The spectral diagram for this case is shown in Figure $1-b$.

Comparing the spectral diagram for $\tau^{1}=0$ with the one for $\tau^{1} \neq 0$, we see that the distribution of spectra is modified but this modification is in such a way as to guarantee Weyl's asymptotic formula for $N(\lambda)$ (Eq. $(4-b)$ ) to hold good. (By folding the diagram in Figure $1-a$ along the $m$-axis, one obtains a diagram which matches the one in Figure $1-b$.)

\subsection{3 $\mathbf{R} P^{2} \# \mathbf{R} P^{2}$ (Klein's bottle).}

The Klein's bottle can be constructed by the point-identification shown in Figure 2.

In mathematical terms, it can be constructed by $\mathbf{R}^{2} / G$ where $G \simeq(\mathbf{Z} \times \mathbf{Z}) \times{ }_{s} \mathbf{Z}_{2}$ $\left(\times_{\mathcal{S}}\right.$ : semi-direct product) [28]. Now, the explicit representation of $G$ will be given: Take $\left(\xi^{1}, \xi^{2}\right)$-space as $\mathbf{R}^{2}$. Let $I=\operatorname{diag}(1,1), B=\operatorname{diag}(1,-1)$ and let $\vec{u}={ }^{t}(1,0)$ and $\vec{v}={ }^{t}(0,1)$ ( $\vec{u}$ and $\vec{v}$ are eigenvectors of $B$ with eigenvalues 1 and -1 respectively). Let $t_{\vec{u}}, t_{\vec{v}}$ represent translations on $\mathbf{R}^{2}$ by $\vec{u}$ and $\vec{v}$, respectively. We choose quantities of the form $\left(A, t_{\vec{a}}\right)$ as a group element $(A=I$ or $B)$ and define a multiple rule as $\left(A, t_{\vec{a}}\right) \cdot\left(A^{\prime}, t_{\vec{a}^{\prime}}\right)=\left(A A^{\prime}, t_{\vec{a}+A \vec{a}^{\prime}}\right)$. Then, $G$ is defined as $G:=\left\{\left(B, t_{\vec{u}}\right)^{m} \cdot\left(I, t_{\vec{v}}\right)^{n} \mid m, n \in \mathbf{Z}\right\}$. By simple manipulations,

$$
\left(B, t_{\vec{u}}\right)^{m} \cdot\left(I, t_{\vec{v}}\right)^{n}=\left(B^{m}, t_{m \vec{u}+(-)^{m} n \vec{v}}\right) .
$$

Here, let us note that $B^{m}=I$ when $m$ is even, and $=B$ when $m$ is odd. Then, this 
element of $G$ acts on any point $\vec{\xi}$ in $\mathbf{R}^{2}$ as $\left(B, t_{\vec{u}}\right)^{m} \cdot\left(I, t_{\vec{v}}\right)^{n} \vec{\xi}=B^{m} \vec{\xi}+m \vec{u}+(-)^{m} n \vec{v}$. If we represent $\left(B^{l}, t_{m \vec{u}+n \vec{v}}\right)$ as $\left(m, n:(-)^{l}\right)(m, n, l \in \mathbf{Z})$, then the multiplication rule reads,

$$
\left(m, n:(-)^{l}\right) \cdot\left(m^{\prime}, n^{\prime}:(-)^{l^{\prime}}\right)=\left(m+m^{\prime}, n+(-)^{l} n^{\prime}:(-)^{l+l^{\prime}}\right)
$$

implying $G \simeq(\mathbf{Z} \times \mathbf{Z}) \times_{\mathcal{S}} \mathbf{Z}_{2}$. Furthermore, $\mathbf{R}^{2} / G$ corresponds to the pointidentification shown in Figure 2, producing Klein's bottle $\mathbf{R} P^{2} \# \mathbf{R} P^{2}$.

The connection of $\left(\xi^{1}, \xi^{2}\right)$ with the standard coordinate $(x, y)$ is the same as in Eq.(8) with the restriction $\tau^{1}=0$ : Because of the particular direction of identification as shown in Figure 2, the deficit angle occurs when $\tau^{1} \neq 0$, contrary to the case of $T^{2}$. Thus we shall investigate only the cases of $\tau^{1}=0$. The eigenvalues become,

$$
\lambda_{m n}=\frac{4 \pi^{2}}{\tau^{2}}\left(\left(\tau^{2}\right)^{2} m^{2}+n^{2}\right),
$$

with normalized eigenfunctions

$$
\begin{aligned}
& 1 \quad \text { for }(m, n)=(0,0) \\
& \sqrt{2} \cos 2 \pi m \xi^{1} \quad \text { for } \quad m \in \mathbf{N}, n=0 \\
& \sqrt{2} \cos 2 \pi n \xi^{2} \quad \text { for } m=0, n \in \mathbf{N} \\
& \left\{\begin{array}{l}
2 \cos 2 \pi m \xi^{1} \cos 2 \pi n \xi^{2} \\
2 \sin 2 \pi m \xi^{1} \cos 2 \pi n \xi^{2}
\end{array} \quad \text { for } \quad m \in \mathbf{N}, n \in \mathbf{N}\right.
\end{aligned}
$$

and

$$
\lambda_{m+1 / 2, n}=\frac{4 \pi^{2}}{\tau^{2}}\left(\left(\tau^{2}\right)^{2}(m+1 / 2)^{2}+n^{2}\right)
$$

with normalized eigenfunctions

$$
\left\{\begin{array}{l}
2 \cos 2 \pi(m+1 / 2) \xi^{1} \sin 2 \pi n \xi^{2} \\
2 \sin 2 \pi(m+1 / 2) \xi^{1} \sin 2 \pi n \xi^{2}
\end{array} \text { for } \quad m \in \mathbf{N}_{0}, n \in \mathbf{N} .\right.
$$

The spectral diagram is shown in Figure 3. Compared with the diagram for $T^{2}$ $\left(\tau^{2}=0\right)($ Figure $1-b)$, the appearance of modes characterized by $m=$ half integer is characteristic. However, the distribution of spectra is again in such a way as to guarantee Weyl's asymptotic formula, $(4-b)$, to hold good. 
We should note that the 2-volume of Klein's bottle constructed in this manner is unity: We can define the integral on a non-orientable manifold $\mathcal{M}$ as half the integral on the double-covering manifold of $\mathcal{M}$. The double-covering manifold of $\mathbf{R} P^{2} \# \mathbf{R} P^{2}$ is $T^{2}$ [11]. Indeed, we can see in Figure 2 the tiles that correspond to $T^{2}$ with 2 -volume being 2 . (For instance, a rectangle defined by points $(0,0)$, $(2,0),(2,1)$ and $(0,1)$.) Thus the 2 -volume of Klein's bottle in our case is 1 .

\subsubsection{The spectral distance between $T^{2}$ and $\mathbf{R} P^{2} \# \mathbf{R} P^{2}$.}

Having obtained spectra for $T^{2}$ and $\mathbf{R} P^{2} \# \mathbf{R} P^{2}$ in previous subsections, we now proceed to calculate the spectral distances for various cases according to Eq.(3) or Eq.(6).

First, let us see how Weyl's asymptotic formula holds nicely for checking the spectra obtained. For instance, the case of $T^{2}$ with Teichmüller parameters $\left(\tau^{1}, \tau^{2}\right)=(0.1,1)$ is shown in Figure 4 and Figure 5. Figure 4 is a $\lambda_{k}-k$ plot. In accordance with $(4-c)(n=2, V=1)$, we see that the inclination of the plot is $4 \pi$. Figure 5 is a $4 \pi N(\Lambda) / V-\Lambda$ plot, which approaches to 1 in accordance with $(4-b)$.

Next, we see the spectral distances $d\left(T^{2}, T^{2}\right)$. Figure $6-a$ indicates the case of $\left(\tau^{1}, \tau^{2}\right)=(0,1)$ and $\left(\tau^{1}, \tau^{2}\right)=(0,2)$ and Figure $6-b$ shows a $d_{\Lambda}((0,1),(0,2))-$ $\Lambda$ plot, giving the spectral distance about 0.219 . The convergence of the scaledependent spectral distance $d_{\Lambda}$ when $\Lambda \rightarrow \infty$ is fairly good.

Figure $7-a$ indicates the case of Klein's bottles with $\left(\tau^{1}, \tau^{2}\right)=(0,10)$ and $\left(\tau^{1}, \tau^{2}\right)=(0,100)$. Figure $7-b$ shows a $d_{\Lambda}((0,10),(0,100))-\Lambda$ plot. The spectral distance is about 2.916 in this case.

Now, the results of calculating the spectral distances between $T^{2}$ and $\mathbf{R} P^{2} \# \mathbf{R} P^{2}$ are quite surprising: They are quite short. For instance, Figure $8-a$ indicates the case of a torus and Klein's bottle with $\left(\tau^{1}, \tau^{2}\right)=(0,1)$ for a torus and $\left(\tau^{1}, \tau^{2}\right)=(0,10)$ for a Klein's bottle, and Figure $8-b$ shows a $d_{\Lambda}-\Lambda$ plot. The spectral distance is about 0.4337 , which is unexpectedly short. 
Figure $9-a, 9-b$ and $9-c$ show some of spectral distances for $T^{2}$, Klein's bottles and mixture cases, respectively. One can see that the spectral distances between $T^{2}$ and $\mathbf{R} P^{2} \# \mathbf{R} P^{2}$ are unexpectedly short. A general tendency is that the spectral distance for $T^{2}-T^{2}$ is longer than the one for $T^{2}-\mathbf{R} P^{2} \# \mathbf{R} P^{2}$, and the same for $\mathbf{R} P^{2} \# \mathbf{R} P^{2}-\mathbf{R} P^{2} \# \mathbf{R} P^{2}$ is the shortest, for fixed parameters $\left(\tau^{1}, \tau^{2}\right)$ and $\left(\tau^{\prime}, \tau^{\prime 2}\right)$. If we employ the density-matrix interpretation (with Caveat $A$ ), these results suggest that an orientable universe and a non-orientable one sometimes interfere with each other quite strongly. In other words, some extra mechanism is needed if they should decohere with each other.

As has already mentioned in advance in $\S \S 2-5$, many examples can be found in Figure $9-a, b, c$ which do not satisfy the triangular inequality, $d\left(\mathcal{G}, \mathcal{G}^{\prime}\right)+$ $d\left(\mathcal{G}^{\prime}, \mathcal{G}^{\prime \prime}\right) \geq d\left(\mathcal{G}, \mathcal{G}^{\prime \prime}\right)$. For instance, in Figure $9-a, d((0,1),(0,500))=68.02$, $d((0,500),(0,1000))=57.45$, while $d((0,1),(0,1000)=137.12 ;$ in Figure $9-c$, $d((0,1),(0,10))=0.4337, d((0,10),(0,100))=2.421$, while $d((0,1),(0,100)=$ 3.488. On the other hand, one example in Figure $9-c$ which satisfies the inequality is for instance, $d((0,1),(0,50))=1.77, d((0,50),(0,100))=2.668$ and $d((0,1),(0,100)=3.488$. The failure of the triangular inequality explicitly indicates that the spectral distance and the DeWitt distance are not equivalent to each other.

At this stage, it is appropriate to compare the spectral distance and the DeWitt distance with each other more explicitly. In the case of $T^{2}$, (the positive-definite sector of) the DeWitt metric reduces to the Poincaré metric on the upper-half plane $\left(\tau^{2}>0\right), G_{A B}=\frac{1}{\tau^{2}} \operatorname{diag}(1,1)$. (As is well-known, one negative signature included in the DeWitt metric corresponds to the conformal deformation in the superspace. We shall come back to this point in §4.) Thus, the DeWitt distance, namely the geodesic distance between $\left(\tau^{1}, \tau^{2}\right)$ and $\left(\tau^{\prime 1}, \tau^{\prime 2}\right)$ w.r.t. the Poincaré metric is [26],[28],

$$
d_{D W}\left(\left(\tau^{1}, \tau^{2}\right),\left({\tau^{\prime}}^{1},{\tau^{\prime}}^{2}\right)\right)=\ln \frac{1+r}{1-r}
$$


where $r=\left|\frac{\tau-\tau^{\prime}}{\tau-\tau^{\prime *}}\right|, \tau=\tau^{1}+i \tau^{2}$ and $\tau^{\prime}=\tau^{\prime 1}+i{\tau^{\prime}}^{2}$. In particular,

$d_{D W}\left(\left(0, \tau^{2}\right),\left(0, \tau^{\prime 2}\right)\right)=\left|\ln \tau^{2} /{\tau^{\prime}}^{2}\right|$, depending only on the ratio $\tau^{2} / \tau^{\prime 2}$. Thus, for instance, $d_{D W}((0,1),(0,2))=d_{D W}((0,10),(0,20))=d_{D W}((0,50),(0,100))=$ $d_{D W}((0,500),(0,1000))=0.693$. (Figure 10). On the other hand, the corresponding spectral distances are $d((0,1),(0,2))=0.219, d((0,10),(0,20))=1.14$, $d((0,50),(0,100))=5.778$ and $d((0,500),(0,1000))=57.45$, which also illustrate the non-equivalence of these two distances.

\section{2. $S^{2}$ AND $\mathbf{R} P^{2}$}

The comparison of the 2 -sphere with the real projective 2 -space $\mathbf{R} P^{2}$ is another case which can be investigated with ease. As has already discussed at the beginning of this section, one can construct the real projective space $\mathbf{R} P^{2}$ of volume $4 \pi R^{2}$ by the antipodal identification on a sphere with radius $\sqrt{2} R$. This space and a sphere with radius $R$ are of same 2 -volume with different orientability as our previous models of tori and Klein's bottles. In the present case, however, the curvatures are also different so that the local geometries are different. Thus, this case serves as the simplest case in which the difference of local geometries as well as the global geometries takes part in. The spectra of $S^{2}$ are

$$
\lambda_{l}=\frac{4 \pi}{V} l(l+1) \quad(\text { multiplicity } 2 l+1, \quad l=0,1,2, \cdots)
$$

where $V$ is the 2-volume of $S^{2}$. The same of $\mathbf{R} P^{2}$ are

$$
\lambda_{l}^{\prime}=\frac{4 \pi}{V} l(2 l+1) \quad(\text { multiplicity } \quad 4 l+1, \quad l=0,1,2, \cdots) .
$$

The spectral distance $d\left(S^{2}, \mathbf{R} P^{2}\right)$ again turns out to be unexpectedly short about 0.8 irrespective of the value of the 2-volume $V$. (See Figure 11.) Figure 12 shows $d\left(S^{2}, S^{2}\right)$ with different 2-volumes. It clearly shows the divergent behavior of the spectral distance when volumes are not identical (see Eq.(5)). Significantly, 
in terms of the scale-dependent spectral distance $d_{\Lambda}$, the degree of difference in volumes is represented by the inclination of the curve of the $d_{\Lambda}-\Lambda$ plot: The larger the difference in volume, the larger the inclination of the $d_{\Lambda}-\Lambda$ plot. In other words, although $d_{\Lambda} \rightarrow \infty$ as $\Lambda \rightarrow \infty$ when volumes are different, the asymptotic behavior of $d_{\Lambda}$ approaching to infinity still contains the information of 'closeness' between volumes.

Even pure-mathematically, it is interesting that some manifolds with different topology (or orientability) show a very short spectral distance between them as compared to other manifolds with identical topology. Furthermore, if one employs the density-matrix interpretation (with Caveat $A$ ) for the spectral distance, it suggests that some universes with different orientabilities do not decohere effectively without any other mechanisms. We shall come back to this point in $\S 4$.

\subsection{EPSTEIN's ZETA AND THETA FUnCTIONS, AND WEYL'S ASYMPTOTIC} FORMULA

It is of some interest to construct and investigate in detail the heat kernel for our models, $T^{2}$ and $\mathbf{R} P^{2} \# \mathbf{R} P^{2}$. In these cases, the heat kernel is expressed nicely in terms of Epstein's theta functions [19],[20],[17]. In such cases, the functional relation for these theta functions derives Weyl's asymptotic formula, and relates directly the expression for the heat kernel in terms of mode-summation, with the one in terms of image-summation [30]. In particular, the case of $\mathbf{R} P^{2} \# \mathbf{R} P^{2}$ (Klein's bottle) is non-trivial and interesting as we shall see below. Furthermore, based on the discussion of the analytic properties of Epstein's zeta functions as meromorphic functions on $\mathbf{C}$, the initial condition for these heat kernels can be analyzed from a different viewpoint.

Thus, let us investigate Epstein's theta and zeta functions for $T^{2}$ and $\mathbf{R} P^{2} \# \mathbf{R} P^{2}$.

\subsubsection{The case of $T^{2}$.}

\footnotetext{
* See Appendix for Epstein's theta and zeta functions.
} 
Let us compute the non-local zeta function, $\zeta(x, y: s):=\sum_{i}^{\prime} \psi_{i}(x) \psi_{i}^{*}(y) \lambda_{i}^{-s}$, for $T^{2}$ of the case $\tau^{1} \neq 0$. The case of $\tau^{1}=0$ goes almost similarly. The final result is identical to the result of the case $\tau^{1} \neq 0$ with a replacement of $Q$ by $Q_{0}$ (see Eqs.(9) and (10)). Using results in 3.1.1 (Eq.(9) and below),

$$
\begin{aligned}
\zeta_{T^{2}}\left(\xi^{1^{\prime}} \xi^{2^{\prime}}, \xi^{1} \xi^{2}: s\right) & =\left(4 \pi^{2}\right)^{-s} Z\left\|\begin{array}{cc}
0 & 0 \\
\Delta \xi^{1} & \Delta \xi^{2}
\end{array}\right\|(Q, s) \\
& =2^{-2 s} \pi^{-1} \frac{\Gamma(1-s)}{\Gamma(s)} Z\left\|\begin{array}{cc}
\Delta \xi^{1} & \Delta \xi^{2} \\
0 & 0
\end{array}\right\|\left(Q^{-1}, 1-s\right)
\end{aligned}
$$

where $\Delta \xi^{1}:=\xi^{1}-\xi^{1^{\prime}}, \Delta \xi^{2}:=\xi^{2}-\xi^{2^{\prime}}$, and $(A 5)$ in Appendix has been used in the last line.

The heat kernel $K(x, y: t)=\sum_{i} \psi_{i}(x) \psi_{i}^{*}(y) \exp -\lambda_{i} t$ becomes

$$
\begin{aligned}
K_{T^{2}}\left(\xi^{1^{\prime}} \xi^{2^{\prime}}, \xi^{1} \xi^{2}: t\right) & =\Theta\left\|\begin{array}{cc}
0 & 0 \\
\Delta \xi^{1} & \Delta \xi^{2}
\end{array}\right\|(Q, 4 \pi t) \\
& =\frac{1}{4 \pi t} \Theta\left\|\begin{array}{cc}
\Delta \xi^{1} & \Delta \xi^{2} \\
0 & 0
\end{array}\right\|\left(Q^{-1}, \frac{1}{4 \pi t}\right), \quad(13-b)
\end{aligned}
$$

where $(A 4)$ has been used in the last line.

It is clear that $(13-b)$ can be written as $\sum_{m, n=-\infty}^{\infty} \frac{1}{4 \pi t} \exp -Q^{-1}\left(m+\Delta \xi^{1}, n+\right.$ $\left.\Delta \xi^{2}\right) / 4 t$, which is an image-summation of the heat kernel on $\mathbf{R}^{2}$. Thus, the functional relation $(A 4)$ guarantees the equivalence between mode-summation $(13-a)$ and image-summation $(13-b)$ [30].

Clearly, $K_{T^{2}}$ satisfies the heat equation, $\Delta_{\vec{\xi}} K\left(\vec{\xi}, \vec{\xi}^{\prime}: t\right)=\partial / \partial t K\left(\vec{\xi}, \vec{\xi}^{\prime}: t\right)$. It is of some interest to clarify the initial condition which $K_{T^{2}}$ should satisfy: From Eq. $(13-b)$,

$$
\begin{aligned}
\lim _{t \downarrow 0} K_{T^{2}}\left(\vec{\xi}, \vec{\xi}^{\prime}: t\right)= & \lim _{t \downarrow 0} \frac{1}{4 \pi t} \exp -Q^{-1}\left(\Delta \xi^{1}, \Delta \xi^{2}\right) / 4 t \\
& +\lim _{t \downarrow 0} \sum_{m, n}{ }^{\prime} \frac{1}{4 \pi t} \exp -Q^{-1}\left(m+\Delta \xi^{1}, n+\Delta \xi^{2}\right) / 4 t
\end{aligned}
$$

The first term is a local contribution while the second term is a non-local one 
coming from the point-identification. The first term is equivalent to $\delta\left(\Delta \xi^{1}\right) \delta\left(\Delta \xi^{2}\right)$. The second term can be written as

$$
\begin{aligned}
& \lim _{t \downarrow 0} \sum_{m, n} ' \frac{1}{4 \pi t} \sum_{k=0}^{\infty} \frac{(-)^{k}}{k !}\left[Q^{-1}\left(m+\Delta \xi^{1}, n+\Delta \xi^{2}\right)\right]^{k} \frac{1}{(4 t)^{k}} \\
& \quad=\lim _{t \downarrow 0} \frac{1}{4 \pi t} \sum_{k=0}^{\infty} \frac{(-)^{k}}{k !} \frac{1}{(4 t)^{k}} Z\left\|\begin{array}{cc}
\Delta \xi^{1} & \Delta \xi^{2} \\
0 & 0
\end{array}\right\|\left(Q^{-1},-k\right)
\end{aligned}
$$

This should vanish because $Z\left\|\begin{array}{l}\vec{g} \\ \vec{h}\end{array}\right\|(Q, s)$ (as a meromorphic function extended onto C) has simple zeros at $s=-1,-2, \cdots$, and furthermore, $s=0$ is also a simple zero when $\vec{g} \notin \mathbf{Z}^{N}$ (see Appendix). Thus $K_{T^{2}}$ satisfies the ordinary initial condition for a heat-kernel. It may be noted that if the $\operatorname{limit}_{\Delta \xi^{1,2} \rightarrow 0}$ is taken before $\lim _{t \downarrow 0}$, the second term behaves as $\lim _{t \downarrow 0} \frac{1}{4 \pi t} \sim \delta(0)$. Thus, $\lim _{\Delta \xi^{1,2} \rightarrow 0} \lim _{t \downarrow 0} \neq$ $\lim _{t \downarrow 0} \lim _{\Delta \xi^{1,2} \rightarrow 0}$ as for the second term, because of the special zero-structure of the zeta function.

From Eq.(13-b), one can easily derive Weyl's asymptotic formula for $\sum_{i} \exp -\lambda_{i} t($ see $(4-a))$,

$$
\begin{aligned}
K_{T^{2}}(t) & =\operatorname{Tr} K_{T^{2}}\left(\vec{\xi}, \vec{\xi}^{\prime}: t\right)=\frac{1}{4 \pi t} \Theta\left\|\begin{array}{ll}
0 & 0 \\
0 & 0
\end{array}\right\|\left(Q^{-1}, \frac{1}{4 \pi t}\right) \\
& =\frac{1}{4 \pi t} \sum_{m, n} \exp -\pi Q^{-1}(m, n) / 4 \pi t=\frac{1}{4 \pi t} \sum_{k=0}^{\infty} \frac{(-)^{k}}{(4 t)^{k} k !} Z\left\|\begin{array}{ll}
0 & 0 \\
0 & 0
\end{array}\right\|\left(Q^{-1},-k\right) \\
& =\frac{1}{4 \pi t},
\end{aligned}
$$

where the zero-structure of $Z\left\|\begin{array}{ll}0 & 0 \\ 0 & 0\end{array}\right\|$ has again been used. Noting $(4-a)$ and below, this result matches the fact that $V=1, \chi\left(T^{2}\right)=0$.

3.3.2 The case of $\mathbf{R} P^{2} \# \mathbf{R} P^{2}$ (Klein's bottle). 
We can proceed in an almost parallel manner as in the case of $T^{2}$. The non-local zeta function becomes,

$$
\begin{aligned}
& \zeta_{\text {Klein }}\left(\xi^{1^{\prime}} \xi^{2^{\prime}}, \xi^{1} \xi^{2}: s\right) \\
& \quad=\frac{1}{2}\left(4 \pi^{2}\right)^{-s}\left\{Z\left\|\begin{array}{cc}
0 & 0 \\
\Delta \xi^{1} & \Delta \xi^{2}
\end{array}\right\|\left(Q_{0}, s\right)+\cos \pi \Delta \xi^{1} Z\left\|\begin{array}{cc}
1 / 2 & 0 \\
\Delta \xi^{1} & \Delta \xi^{2}
\end{array}\right\|\left(Q_{0}, s\right)\right. \\
& \left.\quad+Z\left\|\begin{array}{cc}
0 & 0 \\
\Delta \xi^{1} & \Delta_{+} \xi^{2}
\end{array}\right\|\left(Q_{0}, s\right)-\cos \pi \Delta \xi^{1} Z\left\|\begin{array}{cc}
1 / 2 & 0 \\
\Delta \xi^{1} & \Delta_{+} \xi^{2}
\end{array}\right\|\left(Q_{0}, s\right)\right\}, \quad(15)
\end{aligned}
$$

where $\Delta \xi^{i}:=\xi^{i}-\xi^{i^{\prime}}(i=1,2), \Delta_{+} \xi^{2}:=\xi^{2}+\xi^{2^{\prime}}$. Taking the trace of this expression, the local zeta function becomes,

$$
\begin{array}{r}
\zeta_{\text {Klein }}(s)=\frac{1}{2}\left(4 \pi^{2}\right)^{-s}\left\{Z\left\|\begin{array}{ll}
0 & 0 \\
0 & 0
\end{array}\right\|\left(Q_{0}, s\right)+Z\left\|\begin{array}{cc}
1 / 2 & 0 \\
0 & 0
\end{array}\right\|\left(Q_{0}, s\right)\right. \\
\left.+Z\left\|\begin{array}{c}
0 \\
0
\end{array}\right\|\left(Q_{0}(\cdot, 0), s\right)-Z\left\|\begin{array}{c}
1 / 2 \\
0
\end{array}\right\|\left(Q_{0}(\cdot, 0), s\right)\right\} .
\end{array}
$$

The first terms in (15) and (16) are similar to the case of $T^{2}$, while the second terms originate from the half-integer modes $\lambda_{m+1 / 2, n}$, characteristic for the case of Klein's bottle. The last two terms in (15) and (16) reflect the spatial inhomogeneity of the present model. ( $\Delta_{+} \xi^{2} \rightarrow 2 \xi^{2}$ when $\Delta \xi^{1}, \Delta \xi^{2} \rightarrow 0$, indicating the spatial dependence.)

The heat-kernel for Klein's bottle becomes,

$$
\begin{aligned}
& K_{\text {Klein }}\left(\xi^{1^{\prime}} \xi^{2^{\prime}}, \xi^{1} \xi^{2}: t\right) \\
& \quad=\frac{1}{2} \Theta\left\|\begin{array}{cc}
0 & 0 \\
\Delta \xi^{1} & \Delta \xi^{2}
\end{array}\right\|\left(Q_{0}, 4 \pi t\right)+\frac{1}{2} \cos \pi \Delta \xi^{1} \Theta\left\|\begin{array}{cc}
1 / 2 & 0 \\
\Delta \xi^{1} & \Delta \xi^{2}
\end{array}\right\|\left(Q_{0}, 4 \pi t\right) \\
& \quad+\frac{1}{2} \Theta\left\|\begin{array}{cc}
0 & 0 \\
\Delta \xi^{1} & \Delta_{+} \xi^{2}
\end{array}\right\|\left(Q_{0}, 4 \pi t\right)-\frac{1}{2} \cos \pi \Delta \xi^{1} \Theta\left\|\begin{array}{cc}
1 / 2 & 0 \\
\Delta \xi^{1} & \Delta_{+} \xi^{2}
\end{array}\right\|\left(Q_{0}, 4 \pi t\right)
\end{aligned}
$$

The application of the functional relation $(A 4)$ to the heat-kernel $K_{\text {Klein }}\left(\xi, \xi^{\prime}: t\right),(17-a)$, which has been obtained by the mode-summation, 
provides a non-trivial expression for the heat-kernel corresponding to the pointsummation:

$$
\begin{aligned}
& K_{\text {Klein }}\left(\xi^{1^{\prime}} \xi^{2^{\prime}}, \xi^{1} \xi^{2}: t\right) \\
& =\frac{1}{2} \cdot \frac{1}{4 \pi t}\left\{\Theta\left\|\begin{array}{cc}
\Delta \xi^{1} & \Delta \xi^{2} \\
0 & 0
\end{array}\right\|\left(Q_{0}^{-1}, \frac{1}{4 \pi t}\right)+\cos \pi \Delta \xi^{1} \Theta\left\|\begin{array}{cc}
\Delta \xi^{1} & \Delta \xi^{2} \\
1 / 2 & 0
\end{array}\right\|\left(Q_{0}^{-1}, \frac{1}{4 \pi t}\right)\right. \\
& \left.+\Theta\left\|\begin{array}{cc}
\Delta \xi^{1} & \Delta_{+} \xi^{2} \\
0 & 0
\end{array}\right\|\left(Q_{0}^{-1}, \frac{1}{4 \pi t}\right)-\cos \pi \Delta \xi^{1} \Theta\left\|\begin{array}{cc}
\Delta \xi^{1} & \Delta_{+} \xi^{2} \\
1 / 2 & 0
\end{array}\right\|\left(Q_{0}^{-1}, \frac{1}{4 \pi t}\right)\right\}
\end{aligned}
$$

This reduces to the expression

$$
\begin{aligned}
K_{\text {Klein }}\left(\xi, \xi^{\prime}: t\right) \\
\quad=\sum_{m, n=-\infty}^{\infty} \cos ^{2} \frac{\pi}{2}\left(m+\Delta \xi^{1}\right) \frac{1}{4 \pi t} \exp -Q_{0}^{-1}\left(m+\Delta \xi^{1}, n+\Delta \xi^{2}\right) / 4 t \\
\quad+\sum_{m, n=-\infty}^{\infty} \sin ^{2} \frac{\pi}{2}\left(m+\Delta \xi^{1}\right) \frac{1}{4 \pi t} \exp -Q_{0}^{-1}\left(m+\Delta \xi^{1}, n+\Delta_{+} \xi^{2}\right) / 4 t
\end{aligned}
$$

which is in the form of a point-summation of the heat-kernel for $\mathbf{R}^{2}$ in a non-trivial manner. When $\Delta \xi^{1}=0$, only the terms for $m$ =even (odd) remain in the first (second) summation. Then the first term matches $K_{T^{2}}$ for a torus constructed from $1 \times 2$ band in $\left(\xi^{1}, \xi^{2}\right)$-space, which corresponds to the covering space [11] of our Klein's bottle. The second term originates from the twisting in the pointidentification for constructing the model.

Taking the trace of this, the corresponding theta function becomes,

$$
\begin{aligned}
K_{\text {Klein }}(t):= & \sum_{i} \exp -\lambda_{i} t \\
= & \frac{1}{2} \Theta\left\|\begin{array}{ll}
0 & 0 \\
0 & 0
\end{array}\right\|\left(Q_{0}, 4 \pi t\right)+\frac{1}{2} \Theta\left\|\begin{array}{cc}
1 / 2 & 0 \\
0 & 0
\end{array}\right\|\left(Q_{0}, 4 \pi t\right) \\
& +\frac{1}{2} \Theta\left\|\begin{array}{l}
0 \\
0
\end{array}\right\|\left(Q_{0}(\cdot, 0), 4 \pi t\right)-\frac{1}{2} \Theta\left\|\begin{array}{c}
1 / 2 \\
0
\end{array}\right\|\left(Q_{0}(\cdot, 0), 4 \pi t\right) .
\end{aligned}
$$


The interpretation of each term is similar to the case of the zeta function.

The functional relation helps us to get further insight. By the use of $(A 4)$, $K_{\text {Klein }}(t)$ becomes

$$
\begin{gathered}
K_{\text {Klein }}(t)=\frac{1}{2} \cdot \frac{1}{4 \pi t}\left\{\Theta\left\|\begin{array}{ll}
0 & 0 \\
0 & 0
\end{array}\right\|\left(Q_{0}^{-1}, \frac{1}{4 \pi t}\right)+\Theta\left\|\begin{array}{cc}
0 & 0 \\
1 / 2 & 0
\end{array}\right\|\left(Q_{0}^{-1}, \frac{1}{4 \pi t}\right)\right. \\
\left.+\frac{1}{\sqrt{\tau^{2}}} \Theta\left\|\begin{array}{l}
0 \\
0
\end{array}\right\|\left(Q_{0}(\cdot, 0)^{-1}, \frac{1}{4 \pi t}\right)-\frac{1}{\sqrt{\tau^{2}}} \Theta\left\|\begin{array}{c}
0 \\
1 / 2
\end{array}\right\|\left(Q_{0}(\cdot, 0)^{-1}, \frac{1}{4 \pi t}\right)\right\} \\
=\frac{1}{4 \pi t}
\end{gathered}
$$

where we have followed the same discussion as the case of $K_{T^{2}}(t)$. This result coincides with Weyl's asymptotic formula $(4-a)$ corresponding to our model $(n=$ $2, V=1$, Euler number $=0$ ).

\section{Discussions}

Let us now compare the spectral distance and the DeWitt distance with each other. There are several differences in between them.

First, the DeWitt metric appears within the realm of general relativity, while the spectral distance has been introduced from a general argument $(\S \S 2-1)$, which is itself independent of general relativity.

Secondly, the DeWitt metric is a metric which can be read out from the structure of the 'kinetic term' of the Hamiltonian constraint of general relativity. Just as the geometrical structure of the configuration space of an ordinary mechanical system reflects on the kinetic term, the DeWitt metric reflects the geometrical structure of the superspace. On the other hand, the spectral distance is the measure of the difference in 'sounds' of two universes. In other words, a suitable matter field is used as a probe of local and global geometry of the universe. In the above sense, the DeWitt distance may be called as a 'kinematical distance', while the spectral distance may be called as a 'dynamical distance'. 
Next, by construction, the DeWitt distance can be defined only between universes in the same superspace. The spectral distance can be defined in principle between any kind of universes. As has been discussed in $\S \S 2-3$ and $\S \S 2-4$, the spectral distance requires a cut-off $\Lambda$ for some cases, like in the case of universes with different dimension or different volume. Significantly, even then, the asymptotic behavior of $d_{\Lambda}$ as $\Lambda \rightarrow \infty$ still contains information of the 'closeness' between two universes as has been discussed in $\S \S 3-2$.

The most striking difference is that the triangular inequality holds for the DeWitt distance by construction, while it fails to hold in general for the spectral distance $(\S \S 2-5)$. (Rigorously speaking, therefore, $d\left(\mathcal{G}, \mathcal{G}^{\prime}\right)$ should be called as like a 'measure of closeness', and not a 'distance'.) This fact explicitly demonstrates the non-equivalence between two distances. There is still a possibility of choosing the function $F(x)$ suitably to make $d\left(\mathcal{G}, \mathcal{G}^{\prime}\right)$ satisfy the triangular inequality $(\S \S 2-1)$. Even though the choice of $F(x)=\exp -x$ causes the failure of the triangular inequality, however, it is still distinguished from other possible choices since it derives the density matrix interpretation of the spectral distance $(\S \S 2-2)$.

We have enumerated the differences between the two kinds of distances above. However, we can also draw some interesting parallels between them. Let $\mathcal{G}$ and $\mathcal{G}^{\prime}$ are of the same dimension and topology.

As far as the models investigated in $\S 3$ concerned, both distances well correlate our intuitive notion of 'similar (or different) shapes'. Although the two distances are not equivalent to each other, correlations between them are quite strong.

Another interesting parallelism appears when volumes are different. The DeWitt metric is not a positive definite metric, but it includes one negative signature. This negative signature corresponds to the direction of conformal deformation in the superspace (i.e. the change of volume preserving information of angles). If two geometries $\mathcal{G}$ and $\mathcal{G}^{\prime}$ lie on different surfaces of constant volume in the superspace, therefore, the DeWitt distance cannot be defined between them. Similarly, the spectral distance between $\mathcal{G}$ and $\mathcal{G}^{\prime}$ becomes divergent in this case $(\S \S 2-3)$. Signif- 
icantly, the asymptotic behavior of the scale-dependent spectral distance $d_{\Lambda}\left(\mathcal{G}, \mathcal{G}^{\prime}\right)$ as $\Lambda \rightarrow \infty$ still provides the information of the 'closeness' of volumes $(\S \S 3-2)$. The above observation suggests that the difference in volume seems to be quite different in nature from the other differences in geometry. Combined with the density matrix interpretation, it suggests that the difference in volume causes a very strong decoherence between two universes $(\S \S 2-2)$. This observation also suggests the modified way of comparing two geometries: Separating the information of volume and the conformal geometry as $\mathcal{G}=(V, \tilde{\mathcal{G}})(\operatorname{vol} \tilde{\mathcal{G}}=1)$, a set $\left(V, V^{\prime}\right)$ and $d\left(\tilde{\mathcal{G}}, \tilde{\mathcal{G}}^{\prime}\right)$ may be used as a measure of closeness between $\mathcal{G}$ and $\mathcal{G}^{\prime}$.

Now, let us discuss about the spectral distance between universes with different topologies. To extract the pure topological effects, we have prepared models of $T^{2}$ and Klein's bottles: Both are locally flat and $T^{2}$ corresponds to a double-covering space of Klein's bottle. Thus, they are locally of the same geometry, and the difference between them is purely topological. We have then investigated $d\left(T^{2}, T^{2}\right)$, $d\left(\mathbf{R} P^{2} \# \mathbf{R} P^{2}, \mathbf{R} P^{2} \# \mathbf{R} P^{2}\right)$ and $d\left(T^{2}, \mathbf{R} P^{2} \# \mathbf{R} P^{2}\right)$ with various Teichmüller parameters. As a result, $d\left(T^{2}, \mathbf{R} P^{2} \# \mathbf{R} P^{2}\right)$ has turned out to be quite short compared with $d\left(T^{2}, T^{2}\right)$ and $d\left(\mathbf{R} P^{2} \# \mathbf{R} P^{2}, \mathbf{R} P^{2} \# \mathbf{R} P^{2}\right)$, taking into account that $T^{2}$ and $\mathbf{R} P^{2} \# \mathbf{R} P^{2}$ are topologically different (§§3.1.4).

Furthermore, we have also investigated the cases of $S^{2}$ and $\mathbf{R} P^{2}$ (both are homogeneous and of the same 2 -volume). This time, $S^{2}$ is again a double-covering space of $\mathbf{R} P^{2}$, so that the difference in topology is minimal. In addition to this, local geometries are also different in this case, since (constant) scalar curvatures are different though both of them are homogeneous. (In this sense, the difference in local geometry is also minimal.) As a result, $d\left(S^{2}, \mathbf{R} P^{2}\right)$ has again turned out to be relatively short $(\S \S 3-2)$.

Now, we come back to the original question posed at the beginning of $\S 3$ : Whether universes with different topologies interfere quantum mechanically? A probable answer presented there has been that they decohere with each other strongly since they 'sound' differently, resulting in a long spectral distance. How- 
ever, the above results suggest that this answer is not enough to explain everything. We now know that there are at least some cases in which the spectral distance between two spaces with different orientabilities becomes very short.

Clearly, further investigations are needed to clarify this point. We need to investigate to what extent it is of generality that the spectral distance becomes very short between two spaces $\mathcal{G}$ and $\mathcal{G}^{\prime}$, where $\mathcal{G}=(\Sigma, g), \mathcal{G}^{\prime}=(\Sigma / G, g)$, and $G$ is a discrete subgroup of the isometry group of $(\Sigma, g)$ (like our models of $T^{2}$ and Klein's bottles). We also need to investigate whether the spectral distance between two spaces with more drastic difference in topology becomes large. For instance, the case of two hyperbolic surfaces with a different genus should be investigated. In this case, the properties of spectra ( and the 'length spectra' (a set of lengths of all elementary closed geodesics), which are in some sense the dual concept of the spectra) are extensively investigated by means of Selberg's trace formula [26],[31]. At the same time, in this case, numerical methods are also required to get explicit spectra. This case of hyperbolic surfaces may be an appropriate case as the next step of investigations.

Finally, it is appropriate to mention the relation of the spectral representation with the index theorems [32]. They have some similarity in the sense that both of them connect the eigenvalues of some elliptic operator on a space, with the topological structures of the space. It is clear, however, that the spectral representation provides a finer measure than the index theorems. This can be seen in many respects. For instance, the index theorems talk about the analytical index indD, which is characterized by the zero modes, indD $:=\sum_{j=0}^{m}(-)^{j} \operatorname{dim} k e r \Delta_{j}$, or for the simplest case, $=\operatorname{dim} \operatorname{ker} D-\operatorname{dim} \operatorname{ker} D^{\dagger}[32]$. On the other hand, the spectral representation looks at the whole spectra. This indD takes the value in $\mathbf{Z}$, while the spectral distance varies in $\mathbf{R}$. As is seen in the examples of $\S 3$, for flat tori, the spectral distance even senses the difference in the Teichmüller parameters $\left(\tau^{1}, \tau^{2}\right)$. On the other hand, we can draw many parallels between the discussions of the spectral distance, and those of the index theorems in the context of the anomalies of gauge theories [32]. It is interesting to investigate to what extent these two 
concepts are understood in a unified picture.

Acknowledgements: The author wishes to thank M. Sakagami and S. Jhingan for valuable discussions and encouragements. This work is a part of the IndoJapanese Cooperative Scientific Research Programme by the Japan Society for the Promotion of Science. This work has also been supported by the Yukawa Memorial Foundation, the Japan Association for Mathematical Sciences, and the Japan Society for the Promotion of Science.

\section{APPENDIX}

Here, we shall briefly discuss the basic properties of the theta function and the zeta function due to Epstein.

Let $Q$ be a $N \times N$ symmetric positive definite matrix (so that $\operatorname{det} Q>0$ ). For brevity, let us denote the quadratic form defined by $Q$ as $Q\left(x_{1}, x_{2}, \cdots, x_{N}\right)=Q(\vec{x})$ $:=\left(x_{1}, x_{2}, \cdots, x_{N}\right) Q^{t}\left(x_{1}, x_{2}, \cdots, x_{N}\right)$. The Epstein's theta function and zeta function are defined as [19], respectively,

$$
\begin{gathered}
\Theta\left\|\begin{array}{l}
g_{1} \cdots g_{N} \\
h_{1} \cdots h_{N}
\end{array}\right\|(Q, t):=\sum_{\substack{n_{1}, \cdots, n_{N}=-\infty \\
\times \exp -\pi Q\left(n_{1}+g_{1}, n_{2}+g_{2}, \cdots, n_{N}+g_{N}\right) t,(}}^{\infty} \exp 2 \pi i\left(n_{1} h_{1}+n_{2} h_{2}+\cdots+n_{N} h_{N}\right) \times \\
Z\left\|\begin{array}{l}
g_{1} \cdots g_{N} \\
h_{1} \cdots h_{N}
\end{array}\right\|(Q, s):=\sum_{\substack{n_{1}, \cdots, n_{N}=-\infty \\
\times\left(Q\left(n_{1}+g_{1}, n_{2}+g_{2}, \cdots, n_{N}+g_{N}\right)\right)^{-s},}}^{\infty} \exp 2 \pi i\left(n_{1} h_{1}+n_{2} h_{2}+\cdots+n_{N} h_{N}\right) \times \\
\times(A 2)
\end{gathered}
$$

(For Re $s>N / 2$, with the analytic continuation onto $\mathbf{C}$ )

where the prime on the sigma-symbol in $(A 2)$ indicates that $\left(n_{1}, n_{2}, \cdots, n_{N}\right)=$ $-\left(g_{1}, g_{2}, \cdots, g_{N}\right)$ should be excluded from the summation when $\left(g_{1}, g_{2}, \cdots, g_{N}\right) \in$ $\mathbf{Z}^{N}$, to avoid divergence. Introducing the vector-notation, $(A 1)$ and $(A 2)$ can be expressed as, respectively,

$$
\Theta\left\|\begin{array}{c}
\vec{g} \\
\vec{h}
\end{array}\right\|(Q, t):=\sum_{\vec{n} \in \mathbf{Z}^{N}} \exp 2 \pi i \vec{n} \cdot \vec{h} \exp -\pi Q(\vec{n}+\vec{g}) t
$$




$$
Z\left\|\begin{array}{l}
\vec{g} \\
\vec{h}
\end{array}\right\|(Q, s):=\sum_{\vec{n} \in \mathbf{Z}^{N}} \prime \exp 2 \pi i \vec{n} \cdot \vec{h} \quad Q(\vec{n}+\vec{g})^{-s}
$$

These are related to each other by the Mellin transformation,

$$
\mathbf{M}_{s}\left(\Theta\left\|\begin{array}{l}
\vec{g} \\
\vec{h}
\end{array}\right\|(Q, \cdot)-\delta_{\vec{g}} \exp -i 2 \pi \vec{g} \cdot \vec{h}\right)=\pi^{-s} \Gamma(s) Z\left\|\begin{array}{l}
\vec{g} \\
\vec{h}
\end{array}\right\|(Q, s)
$$

where, $\mathbf{M}_{s} f(\cdot)=\int_{0}^{\infty} d t t^{s-1} f(t)$, and $\delta_{\vec{g}}=1$ when $\vec{g} \in \mathbf{Z}^{N},=0$ otherwise.

They are generalizations of Jacobi's theta function and Riemann's zeta function. (More general definitions than $(A 1)$ and $(A 2)$ are possible [20], but the above are sufficient for our purposes.) Just like Jacobi's theta function and Riemann's zeta function, they satisfy the functional relations, which are very useful for physical applications [30]:

$$
\begin{gathered}
\Theta\left\|\begin{array}{c}
\vec{g} \\
\vec{h}
\end{array}\right\|(Q, t)=\frac{\exp -i 2 \pi \vec{g} \cdot \vec{h}}{\sqrt{\operatorname{det} Q} t^{N / 2} \cdot \Theta\left\|\begin{array}{c}
-\vec{h} \\
\vec{g}
\end{array}\right\|\left(Q^{-1}, \frac{1}{t}\right),} \quad, \quad(A 4) \\
\pi^{-s} \Gamma(s) Z\left\|\begin{array}{l}
\vec{g} \\
\vec{h}
\end{array}\right\|(Q, s)=\pi^{-\left(\frac{N}{2}-s\right)} \Gamma\left(\frac{N}{2}-s\right) \cdot \frac{\exp -i 2 \pi \vec{g} \cdot \vec{h}}{\sqrt{\operatorname{det} Q}} \cdot Z\left\|\begin{array}{c}
-\vec{h} \\
\vec{g}
\end{array}\right\|\left(Q^{-1}, \frac{N}{2}-s\right) .
\end{gathered}
$$

The analytic continuation makes the zeta function expressed as in (A2) a meromorphic function on $\mathbf{C}$. Its pole-structure and zero-structure as a meromorphic function are as follows:

(i) When $\vec{h} \notin \mathbf{Z}^{N}$, it is holomorphic on $\mathbf{C}$. There are simple zeros at least at $s=-1,-2, \cdots$. Furthermore, there occurs one more simple zero at $s=0$ iff $\vec{g} \notin \mathbf{Z}^{N}$.

(ii) When $\vec{h} \in \mathbf{Z}^{N}$, there is a simple pole at $s=N / 2$ with residue $\frac{\pi^{N / 2}}{\Gamma(N / 2)} \frac{1}{\sqrt{\operatorname{det} Q}}$. Simple zeros at least at $s=-1,-2, \cdots$. Furthermore, there occurs one more simple zero at $s=0$ iff $\vec{g} \notin \mathbf{Z}^{N}$. 
Note that the above-mentioned zeros are only the ones which are found from the discussions of analytic properties ('trivial zeros'). Nothing definite can be said for other zeros even for the simplest case, i.e. the case of Riemann's zeta function (corresponding to the case of $Q \equiv 1, \vec{g}=\vec{h}=\overrightarrow{0}$ ) (one may remember the 'Riemann conjecture' [33]).

We now prove (A4). Let us remember the Poisson's summation formula [34],

$$
\sum_{\vec{n} \in \mathbf{Z}^{N}} \psi(\vec{h}+\vec{n})=\sum_{\vec{n} \in \mathbf{Z}^{N}} \exp i 2 \pi \vec{n} \cdot \vec{h} \hat{\psi}(\vec{n})
$$

where $\hat{\psi}$ is the Fourier transformation of $\psi: \hat{\psi}(\vec{k})=\int_{-\infty}^{\infty} \psi(\vec{x}) \exp -i 2 \pi \vec{k} \cdot \vec{x} d \vec{x}$, $\psi(\vec{x})=\int_{-\infty}^{\infty} \hat{\psi}(\vec{k}) \exp i 2 \pi \vec{k} \cdot \vec{x} d \vec{k}$. (Putting the $2 \pi$ in the exponential is just for the neatness of the formulas [34].) Taking $\hat{\psi}(\vec{k})=\exp -\pi Q(\vec{k}+\vec{g}) t$, the R.H.S. of (A6) becomes $\Theta\left\|\begin{array}{l}\vec{g} \\ \vec{h}\end{array}\right\|(Q, t)$, which in turn is the L.H.S. of $(A 4)$. For this choice of $\hat{\psi}$, its inverse Fourier transformation becomes,

$$
\psi(\vec{x})=\frac{\exp -i 2 \pi \vec{g} \cdot \vec{x}}{\sqrt{\operatorname{det} Q} t^{N / 2}} \exp -\pi Q^{-1}(\vec{x}) \frac{1}{t} .
$$

Thus, the L.H.S. of $(A 6)$ turns out to be the R.H.S. of $(A 4)$, which proves $(A 4)$.

Noting $(A 3)$, one basically performs the Mellin transformation of the both sides of (A4) to derive (A5), but a bit of care should be taken for the case of $\vec{g} \in \mathbf{Z}^{N}$. Thus, let us set $\phi\|\| \begin{aligned} & \vec{g} \\ & \vec{h}\end{aligned}\|(Q, t)=\Theta\| \begin{gathered}\vec{g} \\ \vec{h}\end{gathered} \|(Q, t)-\delta_{\vec{g}} \exp -i 2 \pi \vec{g} \cdot \vec{h}$. Then,

$$
\begin{aligned}
\phi\left\|\begin{array}{l}
\vec{g} \\
\vec{h}
\end{array}\right\|(Q, t) & =-\delta_{\vec{g}} \exp -i 2 \pi \vec{g} \cdot \vec{h}+\frac{1}{\sqrt{\operatorname{det} Q} t^{\frac{N}{2}}} \delta_{\vec{h}} \\
& +\frac{1}{\sqrt{\operatorname{det} Q} t^{\frac{N}{2}}} \exp -i 2 \pi \vec{g} \cdot \vec{h} \phi\left\|\begin{array}{c}
-\vec{h} \\
\vec{g}
\end{array}\right\|\left(Q^{-1}, t^{-1}\right)
\end{aligned}
$$

because of $(A 4)$. Then, its Mellin transformation becomes

$$
\mathbf{M}_{s} \phi\left\|\begin{array}{l}
\vec{g} \\
\vec{h}
\end{array}\right\|(Q, \cdot)=\left(\int_{0}^{1}+\int_{1}^{\infty}\right) \phi\left\|\begin{array}{l}
\vec{g} \\
\vec{h}
\end{array}\right\|(Q, t) t^{s-1} d t
$$




$$
\begin{aligned}
= & \int_{1}^{\infty} \phi\|\vec{g}\|(Q, 1 / t) t^{-1-s} d t+\int_{1}^{\infty} \phi\|\vec{g}\| \mid(Q, t) t^{s-1} d t \\
= & \frac{\exp -i 2 \pi \vec{g} \cdot \vec{h}}{\sqrt{\operatorname{det} Q}}\left\{-\frac{\exp -i 2 \pi(-\vec{h}) \cdot \vec{g}}{N / 2-s} \delta_{-\vec{h}}-\frac{1}{\sqrt{\operatorname{det} Q^{-1}}} \frac{1}{(N / 2-s)-N / 2} \delta_{\vec{g}}\right. \\
& +\frac{\exp -i 2 \pi(-\vec{h}) \cdot \vec{g}}{\sqrt{\operatorname{det} Q^{-1}}} \int_{1}^{\infty} \phi\|\vec{g}\| \vec{h} \|(Q, t) t^{N / 2-(N / 2-s)-1} d t \\
& \left.+\int_{1}^{\infty} \phi\|-\vec{h}\|\left(Q^{-1}, t\right) t^{N / 2-s-1} d t\right\} \\
= & \frac{\exp -i 2 \pi \vec{g} \cdot \vec{h}}{\sqrt{\operatorname{det} Q}} \mathbf{M}_{N / 2-s} \phi\|-\vec{h}\|\left(Q^{-1}, \cdot\right) .
\end{aligned}
$$

Here, the change of variable $t \rightarrow 1 / t$ has been made to get the first term in the second line, and $(A 7)$ has been substituted into the same term to get the next line. Then, the same procedure has been repeated to get the last line. Along with $(A 3)$, this proves $(A 5)$.

We can see the pole-structure of the L.H.S. of $(A 3)$, i.e. of $\mathbf{M}_{s} \phi\|\vec{g}\| \vec{h} \|(Q, \cdot)$ in the third line of $(A 8)$. Noting that $\Gamma(s)$ has simple poles at $s=-k(k=0,1,2, \cdots)$ with residue $\frac{(-)^{k}}{k !}$, we see the above-mentioned pole- and zero-structures of $Z\left\|\begin{array}{l}\vec{g} \\ \vec{h}\end{array}\right\|(Q, s)$ from $(A 3)$. 


\section{REFERENCES}

1. See e.g., P.J.E. Peebles, Principles of Physical Cosmology, Princeton University Press, Princeton, 1993.

2. L.Z. Fang and H. Sato, Comm. Theor. Phys. 2, 1055 (1983).

3. A.A. Starobinsky, JETP Lett. 57, 622 (1993); D. Stevens, D. Scott and J. Silk, Phys. Rev. Lett. 71, 20 (1993).

4. M. Seriu, Proceedings of the Indian Association for General Relativity and Gravitation (1994); M. Seriu and T.P. Singh, Phys. Rev. D50, 6165 (1994).

5. J.A. Wheeler, Phys. Rev. 97, 511 (1955).

6. C.W. Misner and J.A. Wheeler, Ann. Phys. 2, 525 (1957); J.A. Wheeler, Geometrodynamics, Academic Press, New York, 1962; R. Sorkin, J. Phys. A10, 717 (1977).

7. e.g. M.S. Morris, K.S. Thorne and U. Yurtsever, Phys. Rev. Lett. 61, 1446 (1988).

8. e.g. G.W. Gibbons and J.B. Hartle, Phys. Rev. D42, 2458 (1990).

9. M. Visser, Phys. Rev. D41, 1116 (1990).

10. M. Seriu, Physics Letters B319, 74 (1993); Vistas in Astronomy 37, 637 (1993).

11. B.A. Dubrovin, A.T. Fomenko and S.P. Novikov, Modern Geometry - Methods and Applications Part II, Springer-Verlag, New York, 1985.

12. J.A. Wheeler, Ann. Phys.(N.Y.) 2, 604 (1957).

13. M. Kac, Am. Math. Mon. 73(4), Part II, 1 (1966).

14. J. Milnor, Proc. Nat. Acad. Sci. USA 51, 542 (1964).

15. M.F. Vignéras, Ann. of Math. 112, 21 (1980); A. Ikeda, J. Math. Soc. Japan 35, 437 (1983). 
16. D. Deturck, H. Gluck, C. Gordon and D. Webb, in Mechanics, Analysis and Geometry: 200 Years after Lagrange, edited by M. Francaviglia, Elsevier Science Publishers B.V., Amsterdam, 1991.

17. T. Sunada, Fundamental Groups and the Laplacian, Kinokuniya Publishing, Tokyo, 1988.

18. B.S. DeWitt, Phys. Rev. 160, 1113 (1967).

19. P. Epstein, Math. Ann. 56, 615 (1903); ibid 63, 205 (1907).

20. C. L. Siegel, Advanced Analytic Number Theory, Tata Institute of Fundamental Research, Bombay, 1980.

21. L.D. Landau and E.M. Lifshitz, Quantum Mechanics (Non-relativistic Theory) 3rd Ed., §41 and §53, Pergamon Press, Oxford, 1977.

22. H.D. Zeh, Phys. Lett. A, 9 (1986).

23. C. Kiefer, Class. Quantum Grav. 4, 1369 (1987).

24. T. Padmanabhan, Phys. Rev. D39 , 2924 (1989).

25. J.J. Halliwell and S.W. Hawking, Phys. Rev. D 31, 1777 (1985).

26. I. Chavel, Eigenvalues in Riemannian Geometry, Academic Press, Orland, 1984.

27. E.B. Davies, Heat Kernels and Spectral Theory, Cambridge University Press, Cambridge, 1989.

28. J. Wolf, Spaces of Constant Curvature, McGraw-Hill, New York, 1967.

29. B. Hatfield, Quantum Field Theory of Point Particles and Strings, Addison -Wesley, Redwood City, California, 1992.

30. J.S. Dowker, J. Math. Phys. 28, 33 (1987); Phys. Rev. D40, 1938 (1989).

31. N.L. Balaza and A. Voros, Phys. Rep. 143, 109 (1986).

32. M. Nakahara, Geometry, Topology and Physics, IOP Publishing, Bristol, 1990. 
33. L.V. Ahlfors, Complex Analysis, McGraw-Hill, New York, 1979.

34. P. Cartier, in From Number Theory to Physics, edited by M. Waldschmidt, P. Moussa, J.-M. Luck and C. Itzykson, Springer-Verlag, Berlin, 1992. 


\section{Figure Captions}

Figure $1-a$ The spectral diagram for $T^{2}\left(\tau^{1} \neq 0\right)$. Symbols 'i' and 'ii' represent the multiplicity 1 and 2, respectively. For instance, the multiplicity of the eigenvalue $\lambda_{(0,0)}$ (zero-mode) is 1 and the same of the eigenvalue $\lambda_{(2,3)}$ is 2 .

Figure $1-b$ The spectral diagram for $T^{2}\left(\tau^{1}=0\right)$. The symbol 'iv' represents multiplicity 4.

Figure 2 Klein's bottle constructed by $\mathbf{R}^{2} / G$. All line segments which are parallel to each other should be identified respecting the direction shown by an arrow sign. A special letter is drawn to visualize the way of identification.

Figure 3 The spectral diagram for Klein's bottle $\mathbf{R} P^{2} \# \mathbf{R} P^{2}$.

Figure 4 A $\lambda_{k}-k$ plot for a torus with $\left(\tau^{1}, \tau^{2}\right)=(0.1,1)$.

Figure 5 A $4 \pi N(\Lambda)-\Lambda$ plot for a torus with $\left(\tau^{1}, \tau^{2}\right)=(0.1,1)$.

Figure $6-a$ Two tori with $\left(\tau^{1}, \tau^{2}\right)=(0,1)$ and $\left(\tau^{1}, \tau^{2}\right)=(0,2)$.

Figure $6-b \mathrm{~A} d_{\Lambda}-\Lambda$ plot for the tori shown in Figure $6-a$.

Figure $7-a$ Two Klein's bottles with $\left(\tau^{1}, \tau^{2}\right)=(0,10)$ and $\left(\tau^{1}, \tau^{2}\right)=(0,100)$.

Figure $7-b$ A $d_{\Lambda}-\Lambda$ plot for Klein's bottles shown in Figure $7-a$.

Figure $8-a$ A torus with $\left(\tau^{1}, \tau^{2}\right)=(0,1)$ (a regular square) and Klein's bottle with $\left(\tau^{1}, \tau^{2}\right)=(0,10)$ (a rectangle)

Figure $8-b \mathrm{~A} d_{\Lambda}-\Lambda$ plot for a torus and Klein's bottle shown in Figure $8-a$.

Figure $9-a$ Spectral distances between two tori. Parameters $\left(\tau^{1}, \tau^{2}\right)$ for two tori and the spectral distance between them are indicated.

Figure 9-b Spectral distances between two Klein's bottles. Parameters $\left(\tau^{1}, \tau^{2}\right)$ for two Klein's bottles and the spectral distance between them are indicated.

Figure $9-c$ Spectral distances between a torus and Klein's bottle. From top to bottom, parameters $\left(\tau^{1}, \tau^{2}\right)$ for a torus and Klein's bottle, and the spectral distance between them are indicated, in this order. 
Figure 10 Distances between two tori defined by the DeWitt metric.

Figure 11 The spectral distance between a 2 -sphere and a real projective space with identical 2-volumes (area).

Figure 12 The spectral distance between two 2-spheres with different 2-volumes, 1.0 and 1.1. 